\title{
BPHZL Renormalization of $1 / N$ Expansion and Critical Behaviour of the Three-Dimensional Chiral Field
}

\author{
I. Ya. Aref'eva, E. R. Nissimov, and S. J. Pacheva \\ Steklov Mathematical Institute, 117333 Moscow, USSR
}

\begin{abstract}
A modified soft mass renormalization scheme for $1 / N$ expansion of the three dimensional $O(N)$-invariant chiral field in the high- and low-temperature phases, as well as at the critical point, is constructed, free of infrared divergencies in each separate diagram. Generalized quantum chirality identities for composite operators are derived, from which the renormalizability of the model follows. The approach formulated here is applied to a rigorous analysis of the universal critical behaviour of the $N$-component chiral field in three dimensions.
\end{abstract}

\section{Introduction}

In this paper we consider Euclidean chiral field $n(x)=\left(n_{1}(x), \ldots, n_{N}(x)\right), x \in \mathbb{R}^{3}$ taking values on the sphere with the simple Lagrangian:

$$
\begin{aligned}
\mathscr{L} & =-\frac{1}{2}\left(\partial_{\mu} n\right)^{2}, \\
n^{2}(x) & =\frac{N \mu}{T},
\end{aligned}
$$

where $n^{2}(x) \equiv(n(x), n(x))=\sum_{\alpha=1}^{N} n_{\alpha}^{2}(x),[\mu$ : mass parameter, $T$ : coupling constant (temperature)].

The chiral field appears in various problems in different (Euclidean) spacetime dimensions $D$. In statistical physics it describes the critical behaviour of classical lattice Heisenberg model of $N$-component spins with $O(N)$ invariant intersection [1]. In field theory it is used in a series of models in the realistic case $D=4, x \in \mathbb{M}^{4}$. The chiral field was first introduced [2] to describe the low-energy $\pi$-meson scattering (non-linear $\sigma$ model). It was applied to construct an unfield model of weak and electromagnetic interactions [3], to introduce mass of the Yang-Mills field in a gauge invariant manner [4], and in models of extended particles [5-7]. Recently there has been an increased interest in the two-dimensional chiral field model $\left(x \in \mathbb{M}^{2}\right.$ or $\left.\mathbb{R}^{2}\right)$ due to the existing similarities between it and non-Abelian 
gauge theories in $D=4$ [8]. The quantum chiral field in $D=2$ was studied quite exhaustively. Its asymptotic freedom was discovered in [8] and renormalizability within the standard perturbation theory in the coupling constant $T$ was proved in $[9,10]$ and of its $1 / N$ expansion was shown in $[11,12]$. Higher local polynomial conserved currents $[13,14]$ as well as nontrivial nonlocal conserved charge [15] were derived and the exact $S$-matrix was constructed [16]. The interesting supersymmetric extension of this model was also examined [17].

The standard perturbation theory in $T$ is nonrenormalizable for $D=3,4[18]^{1}$. A more relevant frame for the three dimensional chiral field theory is the systematic $1 / N$ expansion $[12,21] .1 / N$ expansion was applied to treat various models (see, e.g. [22] and the references quoted therein). The chiral field in the leading order of $1 / N$ was considered in $[9,19]$.

In this model a phase transition of second order takes place: above the critical point the theory is in a symmetrical phase and the correlation functions decrease exponentially, below the critical point $O(N)$ symmetry breaks and long range order appears $[9,19,21,23]$. In $[21,12,24]$ it was proved that there are no ultraviolet (UV) nor infrared (IR) divergencies in both phases and the number of counterterms was shown to be finite. The quantum analogue of classical nonlinearity condition $(n, n)=$ const (called quantum chirality), which is of fundamental importance for the renormalizability was proved in $[12,24]$. Quantum chirality implies that the only necessary renormalizations are coupling constant and wave function renormalizations.

However, the problem of existence of a systematic $1 / N$ expansion directly at the critical point was not solved. This paper is aimed at constructing a systematically renormalized solution for the Green's functions of the chiral field in $D=3$ in each order of $1 / N$, meeting the following requirements :

a) An unified (i.e., suited for both phases as well as for the critical theory) $1 / N$ expansion, free of IR divergencies in each separate Feynman diagram (in the Goldstone phase and critical theory).

b) Each separate diagram in the critical theory is a continuous limit of the corresponding diagram(s) when the mass $m \rightarrow 0$ in the high-temperature phase or the order parameter $f \rightarrow 0$ in the low-temperature phase (i.e., a phase transition of second order occurs).

Our purpose is achieved by applying a modified Bogoliubov-Parasiuk-HeppZimmermann-Lovenstein (BPHZL) renormalization scheme with soft mass and additional IR subtractions $[25,26]$. Analogous approach was introduced and used in conventional perturbation theory of several models with massless particles in $D=4:\left(\varphi^{2}\right)_{4}^{2}[25]$, non-Abelian gauge field in the usual vacuum [27] and instanton [28] sectors; the Goldstons and Higgs models [25]; non-Abelian Higgs-Kibble and Georgi-Glashow models [29]. A beautiful feature of this scheme in that there is no need of investigating the vanishing mass behaviour of the massive theory nor of subtractions at non-zero external momenta.

The renormalization procedure accepted here is characterized by the fact that the UV and IR subtraction degrees $\delta(\lambda)$ and $\varrho(\lambda)$ of an one-particle irreducible

1 Therefore a double expansion in $T$ and $\varepsilon=D-2$ was suggested in [19] and numerical results for the critical exponents in the leading orders [20] were found in good agreement with the experiment 
diagram $\lambda$ are chosen the same in both phases, as well as in the critical theory. Namely, $\delta(\lambda)$ and $\varrho(\lambda)$ are taken equal to their canonical values in the latter. Such a construction is responsible for the successful realization of requirements a) and b).

To prove renormalizability in the scheme with "soft" mass renormalization we derive in the framework of normal products formalism [30] (Sect. 3) the general form of the quantum chirality identities for composite field of form $\left[Q(n, \sigma) n^{2}\right](x)$, $Q(n, \sigma)-O(N)$ invariant monomial in the chiral field $n$ and in the auxiliary field $\sigma$. Thus, in the formal algebra of composite fields, besides the two usual types of linear relations: i) normal product field equations [32,33]; ii) algebraic Zimmermann identities (ZIs) $[30]^{2}$, a new type arises; iii) generalized quantum chirality identities (Sect. 3, Theorem 3.1).

Relations ii) and iii) [together with the Ward-Takahashi identities (WTIs) for the spontaneously broken $O(N)$ symmetry in the low-temperature phase] lead to the following important result : in the set of all (integrated over $x$ ) Euclidean and $O(N)$ invariant (compatible with WTIs in the lower phase) composite operators of canonical UV dimension $\leqq D=3$ there are only two linearly independent ones: $\int d^{3} x \mathscr{N}_{3}^{3}\left[\left(\partial_{\mu} n\right)^{2}\right]_{\otimes}(x)$ and $\int d^{3} x \sigma(x)^{3}$. Only the first of them survives in the critical theory.

This statement is equivalent to the renormalizability of the chiral field in $1 / N$ in the standard BPHZ sense $[18,30]$ : in the effective Lagrangian of the high- and low-temperature phases there are only two arbitrary finite counterterms (the operators listed above), fixed by two physical normalization conditionsnormalization of the $n$-field and mass $m$ (inverse correlation length) or order parameter $f$ (magnetization) normalization condition. As a result, the renormalized Green's functions are parametrized by $m$ or by $f$, respectively, instead of the "temperature" $T$, entering in the intial (nonrenormalized) Lagrangian.

The renormalization group ( $\mathrm{RG}$ ) equations and the WTIs for broken scale invariance are used for the analysis of the scaling behaviour of the chiral field in the critical region, in particular, for the construction of a well defined $1 / N$ expansion of resummed superrenormalizable "temperature" - and "magneticfield" perturbations on the critical theory, free of IR divergences in every separate diagram.

The well known universality relations for critical exponents [35] hold in the present approach. The critical exponents are expressed in terms of only two independent parameters - the anomalous dimensions of the $n$ - and $\sigma$-fields in the critical theory. The numerical results in the leading $1 / N$ order coincide with those for the $O(N)$-invariant lattice Heisenberg model [36].

In earlier works [19] the idea that the chiral field (considered in perturbation theory in $T$ or $1 / N$ together with $\varepsilon=D-2$ expansion) gives a better description of the Goldstone and critical singularities than $O(N)\left(\varphi^{2}\right)_{4-\varepsilon}^{2}$ model $[37,38]$ was suggested and exploited. In [39] another consistent approach to critical behaviour in $1 / N$ frame based on the solution of the Dyson-Schwinger set of equations for

2 In conventional models i), ii) exhaust all independent linear relations in the formal algebra of composite fields $[33,34]$

$3 \mathcal{N}_{\delta}^{\varrho}$ denotes Zimmermann's normal product of UV and IR subtraction degrees $\delta$ and $\varrho$, "®" means that vacuum graphs are omitted. 
the Euclidean noncanonically renormalized Green's functions in massless $O(N)$ invariant $\mathscr{P}\left(\varphi^{2}\right)_{4-\varepsilon}$ models was elaborated.

A parallel of the present approach with [39] is established in [40] where noncanonically renormalized $1 / N$ expansion of massless $O(N)\left(\varphi^{2}\right)_{3}^{2}$ theory is shown to have a well defined critical limit at the IR stable fixed point of the renormalization group. This critical theory coincides with the critical theory of the chiral field. Thus quantum chirality turns out to be an inherent feature of the corresponding universal theory in three dimensions.

The plan of the paper is the following:

In Sect. 2 the modified BPHZL renormalization scheme with "soft" mass is described and the absolute convergence proof for the Green's functions with arbitrary number of composite operators (satisfying the IR convergence criterium) is given.

In Sect. 3 the generalized quantum chirality relations for composite operators are derived.

Section 4 is devoted to the proof of the renormalizability in both phases and at the critical point.

In Sect. 5 the existence of a second order phase transition in the sense of requirement $b$ ) is demonstrated.

In Sect. 6 RG equations and WTIs for broken scale invariance in both phases are derived.

In Sect. 7 we construct IR finite $1 / N$ expansion of "temperature" and "magnetic-field" perturbations on the critical theory and analyze the critical behaviour of the chiral field Green's functions.

A brief review of $1 / N$ graph technique is given in the Appendix.

\section{Absolute Convergence of the Modified Soft Mass Renormalization Scheme in the 1/N Expansion}

The renormalization procedure adopted in the present paper is an extension of Zimmermann-Lowenstein soft mass subtraction scheme for massless theories $[25,26]$ to the case of (Euclidean) Feyman integrals containing unconventional propagators, i.e., those of the auxiliary $\sigma$-field (A.5) and (A.6), which are irrational and transcendental functions of momenta and masses. The softness parameter $s$ $(0 \leqq s \leqq 1)$ is introduced according to the rule: $m \rightarrow m(s) \equiv s m+(1-s) \mu, f^{2} \rightarrow s f^{2}$ ( $\mu$-mass parameter). The main reason for the removal of IR divergencies turns out to be the favourable low-momentum behavior of the $\sigma$-propagator [cf. (A.7)].

We shall essentially use the notations and concepts of $[41,25,26]$. Following general definitions (see, e.g., $[26,42]$ ), the canonical UV and IR degrees, $d(\gamma)$ and $r(\gamma)$ of a one-particle irreducible $(1 P I)$ with respect to $n$ - and $\sigma$-lines graph $\gamma \equiv \gamma_{\left(L_{n}, L_{\sigma}\right)}^{\{P a}$ are written in the form:

$$
\begin{aligned}
& d(\gamma)=3-\bar{d}_{n} L_{n}(\gamma)-\bar{d}_{\sigma} L_{\sigma}(\gamma)+\sum_{\mathbf{v} \in \mathscr{V}(\gamma)}\left(\bar{d}_{\mathbf{v}}-3\right), \\
& r(\gamma)=3-\underline{d}_{n} L_{n}(\gamma)-\underline{d}_{\sigma} L_{\sigma}(\gamma)+\sum_{\mathbf{V} \in \mathscr{V}(\gamma)}\left(\underline{d}_{\mathbf{V}}-3\right) .
\end{aligned}
$$


Here the following notations are used: $\gamma \equiv \gamma_{\left(L_{n}, L_{\sigma}\right)}^{\left\{P_{a}\right.}-1$ PI diagram with $L_{n}(\gamma)$ and $L_{\sigma}(\gamma)$ external $n$ - and $\sigma$-lines; $\mathscr{V}(\gamma)=\mathscr{V}_{\mathscr{L}}(\gamma) \bigcup\left\{\mathbf{V}\left(P_{a}\right)\right\}$-set of all vertices of $\gamma: \mathscr{V}_{\mathscr{L}}(\gamma)$ $=\mathscr{V}_{\sigma n^{2}} \bigcup \mathscr{V}_{\sigma\left(n, F s^{1 / 2}\right.}$-set of Lagrangian vertices, $\mathbf{V}(P)$-vertex of the composite operator $P \equiv P(n, \sigma)(x)$ :

$$
P[n, \sigma](x)=(1-s)^{c} s^{h} \prod_{i=1}^{v_{n}(P)}\left(\partial_{(\mu)_{i}}^{k_{i}} n\right)(x) \prod_{j=1}^{v_{\sigma}(P)}\left(\partial_{(\mu)_{j}}^{p_{j}} \sigma\right)(x), \partial_{(\mu)}^{k} \equiv \prod_{l=1}^{k} \partial / \partial x_{\mu_{l}},
$$

where $v_{n, \sigma}(P)$ is the number of $n, \sigma$-fields in $P, c, h$-positive integers. The canonical UV and IR dimensions of $P, \bar{d}_{P}$ and $\underline{d}_{P}$, are defined as follows:

$$
\begin{aligned}
& \bar{d}_{P}=c+h+\sum_{i=1}^{v_{n}(P)} k_{i}+\sum_{j=1}^{v_{\sigma}(P)} p_{j}+\bar{d}_{n} v_{n}(P)+\bar{d}_{\sigma} v_{\sigma}(P), \\
& \underline{d}_{P}=c+\sum_{i=1}^{v_{n}(P)} k_{i}+\sum_{j=1}^{v_{\sigma}(P)} p_{j}+\underline{d}_{n} v_{n}(P)+\underline{d}_{\sigma} v_{\sigma}(P) .
\end{aligned}
$$

The canonical UV and IR dimensions of the $n$-, $\sigma$-field, $\bar{d}_{n, \sigma}$ and $\underline{d}_{n, \sigma}$, in accordance with the large- and low-momentum behaviour of their propagators (A.7), are given by:

$$
\bar{d}_{n}=\underline{d}_{n}=1 / 2, \quad \bar{d}_{\sigma}=2, \quad \underline{d}_{\sigma}=\left\{\begin{array}{l}
5 / 2 \\
2
\end{array} .\right.
$$

[The relation for $\underline{d}_{\sigma}$ in (2.5) refers to the Goldstone phase and the critical theory, respectively.] When only Lagrangian vertices are present in

$$
\gamma \equiv \gamma_{\left(L_{n}, L_{\sigma}\right)}\left(\bar{d}_{n^{2} \sigma}=\bar{d}_{\sigma\left(n, F s^{1 / 2}\right)}=\underline{d}_{\sigma\left(n, F s^{1 / 2}\right)}=3, d_{n^{2} \sigma}=\left\{\begin{array}{l}
7 / 2 \\
3
\end{array}, \text { resp. }\right)\right.
$$

(2.1) and (2.2) simplify to:

$$
\begin{aligned}
& d(\gamma)=3-1 / 2 L_{n}(\gamma)-2 L_{\sigma}(\gamma) \\
& r(\gamma)= \begin{cases}d(\gamma)+\left|\mathscr{L}_{\sigma}(\gamma)\right|-1 / 2 L_{F}(\gamma)>d(\gamma) & \text { (low-temperature phase) } \\
d(\gamma) & \text { (critical theory) }\end{cases}
\end{aligned}
$$

where $L_{F}(\gamma) \equiv\left|\mathscr{V}_{\sigma\left(n, F s^{1 / 2}\right)}(\gamma)\right|$ is the number of external (blind) $F$-lines, $\mathscr{L}_{n, \sigma}(\gamma)$ - the set of internal $n$-, $\sigma$-lines of $\gamma,\left|\mathscr{L}_{n, \sigma}(\gamma)\right|$ - their number.

It will be necessary to introduce noncanonical UV and IR subtraction degrees, $\delta(\gamma)$ and $\varrho(\gamma)$, constrained by the consistency conditions $[26,42]$ :

$$
\delta(\gamma) \geqq d(\gamma), \delta(\gamma) \geqq d(\gamma /\{\lambda\})+\sum_{i=1}^{c} \delta\left(\lambda_{i}\right) ; \varrho(\gamma) \leqq r(\gamma), \varrho(\gamma) \leqq r(\gamma /\{\lambda\})+\sum_{i=1}^{c} \varrho\left(\lambda_{i}\right),
$$

where $\{\lambda\} \equiv\left\{\lambda_{1}, \ldots, \lambda_{c}\right\}$ is an arbitrary set of nontrivial, mutually disjoint 1PI subdiagrams of $\gamma$ and $\gamma /\{\lambda\}$ represents the corresponding reduced graph.

The concepts of UV and IR degrees and formulae (2.1), (2.2), (2.1'), (2.2'), and (2.6) are immediately generalized for an arbitrary connected graph.

Let us consider connected Green's functions of the general type:

$$
\begin{aligned}
& \left\langle\prod_{a=1}^{A} Q_{a} \prod_{b=1}^{B} \mathcal{N}_{\delta_{b}}^{\varrho_{b}}\left[P_{b}(n, \sigma)\right]\left(x_{b}\right) X_{n} X_{\sigma}\right\rangle, X_{n}=\prod_{i=1}^{L_{n}} n_{\alpha_{i}}\left(x_{i}^{\prime}\right), X_{\sigma}=\prod_{j=1}^{L_{\sigma}} \sigma\left(x_{j}^{\prime \prime}\right), \\
& Q_{a} \equiv \int d^{3} x \mathcal{N}_{\delta_{a}}^{\varrho_{a}}\left[Q_{a}(n, \sigma)\right](x),
\end{aligned}
$$



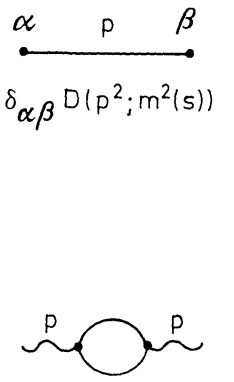

$N S\left(p^{2} ; m^{2}(s)\right)$

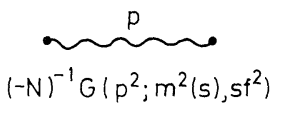

a

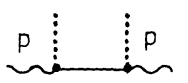

$N s f^{2} D\left(p^{2} ; m^{2}(s)\right)$

b

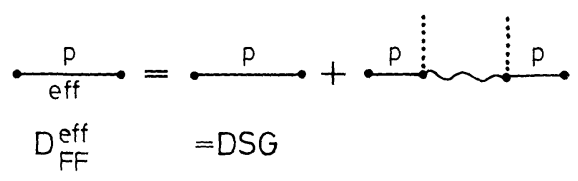

d
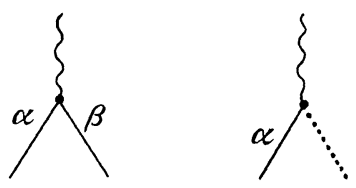

$-\delta \alpha \beta$

$-F_{\alpha} s^{1 / 2}$
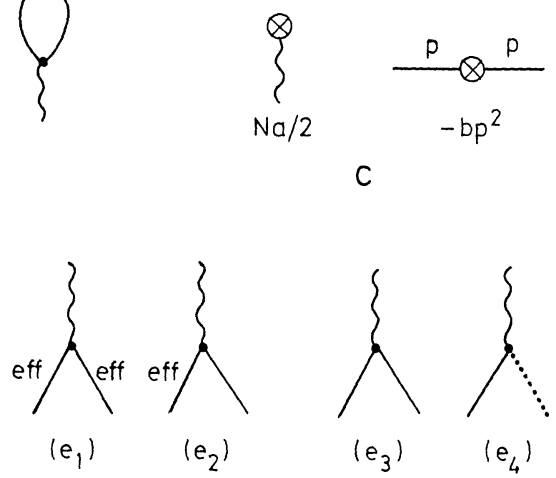

e

Fig. 1. a Graphical elements of $1 / N$ expansion; $\mathbf{b}$ "forbidden" subgraphs for ordinary Green's functions $\left\langle X_{n} X_{\sigma}\right\rangle$; $\mathbf{c}$ finite counterterms vertices; d effective free $n_{F}$-propagator (to lowest order in $1 / N$ ); e effective Lagrangian vertices in the Goldstone phase (after effective summation in $n_{F}$-lines)

where $\varrho_{a} \leqq \underline{d}_{Q_{a}}, \varrho_{b} \leqq \underline{d}_{P_{b}}$ and $\delta_{a} \geqq \bar{d}_{Q_{a}}, \delta_{b} \geqq \bar{d}_{P_{b}}$ are (noncanonical) IR and UV indices of $Q_{a}$ and $P_{b}$. The renormalized $1 / N$ expansion of $(2.7)$ is constructed according to the rules of the $1 / N$ diagram technique (Appendix A, Fig. 1) by means of the Zimmermann's forest formula $[41,25,26]$, attributing to every connected graph $G \equiv G_{\left(L_{n}, L_{\sigma}\right)}^{\left\{P_{3}\right.}$ of $(2.7)$ the expression:

$$
\begin{aligned}
\mathscr{R}_{G}\left(\left\{p^{G}\right\}\right) & =\int \prod_{l} \frac{d^{3} k_{l}^{G}}{(2 \pi)^{3}} R_{G}\left(\left\{p^{G}\right\},\left\{k^{G}\right\}\right) \\
R_{G}\left(\left\{p^{G}\right\},\left\{k^{G}\right\}\right) & =S_{G} \sum_{U \in \mathscr{F} G} \prod_{\gamma \in U}\left(-\tau_{\gamma}^{\delta(\gamma), \varrho(\gamma)} S_{\gamma}\right) I_{G}(U) \equiv\left(1-\tau_{G}^{\delta(G), \varrho(G)}\right) \bar{R}_{G} .
\end{aligned}
$$

In (2.8) $\mathscr{F}_{G}$ is the set of all $G$-forests $U, I_{G}(U)$ is the unrenormalized integrand, in which the momentum of each line $l$ is a linear combination of $\left\{p^{\lambda}\right\},\left\{k^{\lambda}\right\}$, a suitably chosen basis of external and internal momenta of the smallest element $\lambda$ of $U \bigcup\{G\}$ containing $l ; S_{\gamma}$ are substitution operators corresponding to $U$. The subtraction operators attached to every 1 PI graph $\gamma \equiv \gamma_{\left(L_{n}, L_{\sigma}\right)}^{\{P a}$ are defined as follows [26]:

$$
\tau_{\gamma} \equiv \tau_{\gamma}^{\delta(\gamma), \varrho(\gamma)}=t_{p^{\gamma}, s^{\gamma}}^{\delta(\gamma)}+t_{p^{\gamma}, s^{\gamma}-1}^{\varrho(\gamma-1}\left(1-t_{p^{\gamma}, s^{\gamma}}^{\delta(\gamma)}\right)=t_{p^{\gamma}, s^{\gamma}-1}^{\varrho(\gamma)-1}+\left(1-t_{p^{\gamma}, s^{\gamma}-1}^{\varrho(\gamma)-1}\right) t_{p^{\gamma}, s^{\gamma}}^{\delta(\gamma)},
$$

where $t_{x, y}^{d}$ is the usual Taylor subtraction operator, and $\delta(\gamma), \varrho(\gamma)$ satisfy the inequelities (2.6). A concrete choice for $\delta(\gamma), \varrho(\gamma)$ will be made later. 
According to the combined power counting theorem [43] the absolute convergence of $\mathscr{R}_{G}\left(\left\{p^{G}\right\}\right.$ ) (for nonexceptional momenta $\left\{p^{G}\right\}$ in the Goldstone phase and in the critical theory) is equivalent to the criteria:

$$
\begin{aligned}
& {\overline{\operatorname{deg}_{u}}}_{G}\left(\left\{p^{G}\right\},\left\{k^{G}\left(\{u\},\{v\},\left\{p^{G}\right\}\right)\right\}\right)+\operatorname{dim} H<0, \\
& \underline{\operatorname{deg}}_{u} R_{G}\left(\left\{p^{G}\right\},\left\{k^{G}\left(\{u\},\{v\},\left\{p^{G}\right\}\right)\right\}\right)+\operatorname{dim} H>0,
\end{aligned}
$$

where $H$ is an arbitrary hyperplane in the space of the internal momenta defined in a suitable parametrization $k^{G}=k^{G}\left(\{u\},\{v\},\left\{p^{G}\right\}\right)$ as $v_{j}=$ const, $v_{j} \in\{v\}$ and $\overline{\operatorname{deg}}_{x} f(x, y), \operatorname{deg}_{x} f(x, y)$ denote upper and lower degrees of a function $f(x, y)$ with respect to the variable $x$ [43].

Let us introduce a class $\mathscr{Y}$ of functions of momenta and of $s(0 \leqq s \leqq 1)$ having the form:

$$
\begin{aligned}
A(\{p\},\{u\},\{v\} ; s)\left[D_{1}(\{p\},\{u\},\{v\} ; s) D_{2}(\{u\},\{v\})\right]^{-1}, \\
A(\{p\},\{u\},\{v\} ; s) \\
=\sum_{\alpha, \beta, \gamma} \mathscr{P}_{\{\alpha, \beta, \gamma\}}(\{p\},\{u\},\{v\} ; s) \prod_{\alpha}\left[\mathscr{G}^{-1}\left(l_{\alpha}(\{p\},\{u\},\{v\}) ; m(s), s f^{2}\right)\right]^{k_{\alpha}} \\
\quad \cdot \prod_{\beta}\left[\mathscr{G}^{-1}\left(l_{\beta}(\{u\},\{v\}) ; m, f^{2}\right)\right]^{k_{\beta}} \prod_{\gamma}\left[\mathscr{G}^{-1}\left(l_{\gamma}(\{u\},\{v\}) ; \mu, 0\right)\right]^{k_{\gamma}} \\
\equiv \sum_{\alpha, \beta} P_{\alpha \beta} A_{\alpha} B_{\beta}, \\
D_{1}(\{p\},\{u\},\{v\} ; s) \\
=\prod_{\alpha}\left[\mathscr{D}^{-1}\left(l_{\alpha}(\{p\},\{u\},\{v\}) ; m(s)\right)\right]^{n_{\alpha}} \prod_{\beta}\left[\mathscr{G}^{-1}\left(l_{\beta}(\{p\},\{u\},\{v\}) ; m(s), s f^{2}\right)\right]^{n_{\beta}}, \\
D_{2}(\{u\},\{v\}) \\
=\prod_{i}\left[\mathscr{D}^{-1}\left(l_{i}\left(\{u\},\{v\} ; c_{i} \mu\right)\right]^{a_{t}} \prod_{j}\left[\mathscr{D}^{-1}\left(l_{j}(\{u\},\{v\}) ; c_{j} m\right)\right]\right. \\
\quad \cdot \prod_{a}\left[\mathscr { G } ^ { - 1 } ( l _ { a } ( \{ u \} , \{ v \} ; m , f ^ { 2 } ) ] ^ { n _ { a } } \prod _ { b } \left[\mathscr{G}^{-1}\left(l_{b}(\{u\},\{v\} ; \mu, 0)\right]^{n_{b}},\right.\right.
\end{aligned}
$$

where $\mathscr{P}_{\{\alpha, \beta, \gamma\}}$ are polynomials $; k_{\alpha}, k_{\beta}, k_{\gamma}, n_{\alpha}, n_{\beta}, n_{a}, n_{b}, a_{i}, b_{i}$-positive integers; $c_{i}, c_{j}$ take values $0,1,2 ; l(\{p\},\{u\},\{v\})$ is a linear combination of $\{p\},\{u\},\{v\}, \mathscr{D}$, and $\mathscr{G}$ are the propagators of the $n$ - and $\sigma$-fields (A.4)-(A.6). The class $\mathscr{Y}$ has the following properties:

i) $\mathscr{Y}$ is invariant under multiplication by and differentiation in $\{p\}$ and $s$.

ii) Each element of $\mathscr{Y}$ has power asymptotic behaviour in $|l(\{p\},\{u\},\{v\})|$ for large and small momenta $\{p\},\{u\}$, with coefficients which are polynomials in $s$ or $s-1$.

The latter is due to the specific form of the $\sigma$-propagator for $D=3$ (A.5) and (A.6):

$$
8 \pi \mid l\left[\left[\operatorname{arctg} \frac{|l|}{2 m(s)}\right]^{-1}=16|l|+\frac{64}{\pi} m(s)+\left(\frac{16}{\pi}\right)^{2} \frac{m^{2}(s)}{|l|}+\mathcal{O}\left(\left.|l|\right|^{-2}\right) .\right.
$$


It is convenient for the IR convergence to use the notion of augmented graph $\hat{G}$, corresponding to $G[26]$. $\hat{G}$ is formed from $G$ by adding auxiliary fictitious lines, starting at the external vertices of $G$ and all ending at a new vertex $\mathbf{V}_{0}$.

Theorem 2.1. (cf. $[44,26])$. a) Suppose that for every set $\{\gamma\}=\left\{\gamma_{1}, \ldots, \gamma_{c}\right\}$ of mutually disjoint, nontrivial $1 P I \gamma_{i} \subset \hat{G},\{\gamma\} \not \hat{G}$ the IR convergence criterium is satisfied:

$$
v(\hat{G} /\{\gamma\})+\sum_{i=1}^{c} \max \left\{0, \varrho\left(\gamma_{i}\right)\right\}>0 .
$$

Then $\int \pi d p^{G} d k^{G} R_{\hat{G}}\left(\left\{p^{G}\right\},\left\{k^{G}\right\}, s\right)$ is absolutely convergent for every $s \in[0,1]$.

b) Let $\Gamma$ be a $1 P I$ graph such that for every set $\{\gamma\}$ defined in a), for which $\hat{\Gamma} /\{\gamma\}$ does not contain any fictitious line of $\Gamma$, the IR convergence criterium (2.13) is satisfied. Then $\mathscr{R}_{\Gamma}\left(\left\{p^{\Gamma}\right\}, s\right)=\int \prod d k^{\Gamma} R_{\Gamma}\left(\left\{p^{\Gamma}\right\},\left\{k^{\Gamma}\right\}, s\right)$ is absolutely convergent (for nonexceptional $\left\{p^{\Gamma}\right\}$ in the low-temperature phase and in the critical theory).

The proof relies on the concept of forests $U_{H} \in \mathscr{F}_{G}$ (or $U_{H} \in \mathscr{F}_{T}$ ), complete with respect to a hyperplane $H$ (in the space of internal momenta of $G$ (or $\Gamma$ ) and on the decomposition [41]:

$$
R_{G}=\sum_{U_{H}} R_{G}\left(U_{H}\right), R_{G}\left(U_{H}\right)=\left(1-\tau_{G}^{\delta(G), \varrho(G)}\right) Y_{G}\left(U_{H}\right)
$$

with the recursive definition of $Y_{\gamma}\left(U_{H}\right), \gamma \cong G$ :

$$
Y_{\gamma}\left(U_{H}\right)=I_{\bar{\gamma}\left(U_{H}\right)} S_{\gamma} \prod_{j} f_{\gamma_{j}} Y_{\gamma_{j}}\left(U_{H}\right), f_{\gamma_{j}}= \begin{cases}1-\tau_{\gamma_{j}}, & \text { if } \bar{\gamma}_{j}\left(U_{H}\right) \chi H, \bar{\gamma}_{j}\left(U_{H}\right) \| H \\ -\tau_{\gamma_{j}}, & \text { otherwise, }\end{cases}
$$

where $\bar{\gamma}\left(U_{H}\right)=\gamma /\left\{\gamma_{1}, \ldots, \gamma_{c}\right\}, \gamma_{i} \subset \gamma$-maximal in $U_{H}$, and the symbols $\bar{\gamma}\left(U_{H}\right) \chi H$, $\bar{\gamma}\left(U_{H}\right) \| H$ mean that $\bar{\gamma}$ is constant, respectively, variable with respect to $H$ (see [41]). Let us note, that all $Y_{\gamma}\left(U_{H}\right)(2.15)$ belong to the class $\mathscr{Y}(2.11)$. The purely combinatorial part of the proofs of the UV and IR convergence criteria (2.10a) and $(2.10 \mathrm{~b})$ is readily adapted from that of $[44,26]$ to the present case, provided we have proved the following inequalities:

$$
\begin{aligned}
& \overline{\operatorname{deg}}_{u, p^{\gamma}, s^{\gamma}}\left[\tau_{\gamma} Y_{\gamma}\right] \leqq \overline{\operatorname{deg}}_{u, p^{\gamma}, s^{\gamma}} Y_{\gamma}, \bar{\gamma}\left(U_{H}\right) \| H, \\
& \overline{\operatorname{deg}}_{u}\left[\left(1-\tau_{\gamma}\right) Y_{\gamma}\right] \leqq \overline{\operatorname{deg}}_{u, p^{\gamma}, s^{\gamma}} Y_{\gamma}-\max \{\delta(\gamma), \varrho(\gamma)-1\}-1, \bar{\gamma}\left(U_{H}\right) \| H, \\
& \overline{\operatorname{deg}}_{u}\left[\tau_{\gamma} Y_{\gamma}\right] \leqq \overline{\operatorname{deg}}_{u} Y_{\gamma}, \bar{\gamma}\left(U_{H}\right) \chi H \text {. } \\
& \overline{\operatorname{deg}}_{u, p^{\gamma}, s^{\gamma}}\left[\tau_{\gamma} Y_{\gamma}\right] \leqq \overline{\operatorname{deg}}_{u} Y_{\gamma}+\max \{\delta(\gamma), \varrho(\gamma)\}, \bar{\gamma}\left(U_{H}\right) \chi H, \\
& \left.\underline{\operatorname{deg}}_{u, p^{\gamma}, s^{\gamma}-1}\left[\tau_{\gamma} Y_{\gamma}\right] \geqq \min \left\{\underline{\operatorname{deg}}_{u, p^{\gamma}, s^{\gamma}-1} Y_{\gamma}, \underline{\operatorname{deg}}_{u} Y_{\gamma}\right\}+\max (0, \varrho(\gamma))\right\} \text {, }
\end{aligned}
$$

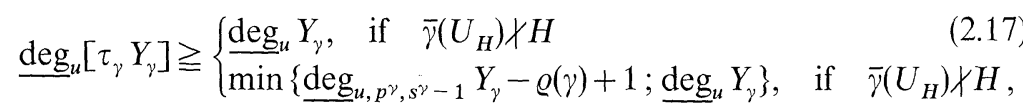

$$
\begin{aligned}
& \underline{\operatorname{deg}}_{u, p^{\gamma}, s^{\gamma-1}}\left[\left(1-\tau_{\gamma}\right) Y_{\gamma}\right] \geqq \underline{\operatorname{deg}}_{u} Y_{\gamma}+\max \{0, \varrho(\gamma)\}, \quad \text { if } \quad \bar{\gamma}\left(U_{H}\right) \nmid H .
\end{aligned}
$$

(2.16) and (2.17) are directly verified using properties i) and ii) of $\mathscr{Y}$ and properties of the subtraction operator $\tau_{\gamma}$ (2.9) (cf. Sect. 3 of [44] and Lemma 3.2 of [26]). 
Theorem 2.2. Euclidean Green's functions (2.7) exist in each order of the soft mass renormalized $1 / N$ expansion, provided $d_{Q_{a}} \geqq 3$ (in the Goldstone phase and in the critical theory).

Proof. As follows from Theorem 2.1 we have to verify (2.13) for each connected graph $G$. When the new vertex $\mathbf{V}_{0}$ belongs to some $\gamma_{j} \in\left\{\gamma_{i}\right\}_{i=0}^{c}$ we assume for definiteness, that it is $\gamma_{0}$. From (2.2) and (2.4b), after simple computation, we obtain the following expression for $r(\Lambda), \Lambda \equiv \hat{G} /\{\gamma\}$ :

$$
\begin{aligned}
r(\Lambda) & =\sum_{\mathscr{V} \mathscr{L}(\Lambda)}\left(\underline{d}_{\mathscr{L}}-3\right)+\sum_{\mathbf{v}\left(Q_{a}\right) \in \mathscr{V}(\Lambda)}\left(\underline{d}_{Q_{a}}-3\right)+\sum_{\mathbf{v}\left(P_{b}\right) \in \mathscr{V}(\Lambda)} \underline{d}_{p_{b}}+3\left\{B\left(\gamma_{0}\right)-\left|\mathscr{L}_{B}^{q}\left(\gamma_{0}\right)\right|\right\} \\
& +\left(3-\underline{d}_{n}\right)\left\{L_{n}(G)-\left|\mathscr{L}_{n}^{q}\left(\gamma_{0}\right)\right|\right\}+\left(3-\underline{d}_{\sigma}\right)\left\{L_{\sigma}(G)-\left|\mathscr{L}_{\sigma}^{q}\left(\gamma_{0}\right)\right|\right\}+\underline{d}_{n} L_{n}\left(\gamma_{0}\right)+\underline{d}_{\sigma} L_{\sigma}\left(\gamma_{0}\right) \\
& -\sum_{j=1}^{c}\left\{3-\underline{d}_{n} L_{n}\left(\gamma_{j}\right)-\underline{d}_{\sigma} L_{\sigma}\left(\gamma_{j}\right)-3 B\left(\gamma_{j}\right)\right\}+r_{0}, \quad r_{0}=\left\{\begin{array}{l}
3, \mathbf{V}_{0} \notin \gamma_{0} \\
0, \mathbf{V} \in \gamma_{0},
\end{array}\right.
\end{aligned}
$$

where $\underline{d}_{\mathscr{L}}=\underline{d}_{\sigma n^{2}}, \underline{d}_{\sigma\left(n, F s^{1 / 2}\right)}$ are the canonical IR dimensions of the Lagrangian vetices $\mathscr{V}_{\mathscr{L}}(\Lambda) ; B(\gamma)$ is the number of composite operator vertices $\mathbf{V}\left(P_{b}\right)$ belonging to $\mathscr{V}(\gamma) ; \mathscr{L}_{n, \sigma, B}^{q}(\gamma)$ denotes the auxiliary fictitious $n$-, $\sigma-, P_{B}$-lines which are internal for $\gamma_{0}$, if $V_{0} \in \gamma_{0} ;\left|\mathscr{L}_{n, \sigma, B}^{q}\left(\gamma_{0}\right)\right|$ - their number, $\left|\mathscr{L}_{n, \sigma, B}^{q}\left(\gamma_{0}\right)\right| \equiv 0$ if $\mathbf{V}_{0} \notin \gamma_{0}$.

Let us first consider the critical theory. In accordance with (2.2) and (2.6), we choose $\left(\underline{d}_{n}=\frac{1}{2}, \underline{d}_{\sigma}=2\right)$ :

$$
\begin{aligned}
\varrho\left(\gamma_{j}\right)= & 3-\frac{1}{2} L_{n}\left(\gamma_{j}\right)-2 L_{\sigma}\left(\gamma_{j}\right) \\
& +\sum_{\mathbf{v}\left(Q_{a}\right) \in \mathscr{V}(\gamma)}\left(\varrho_{a}-3\right)+\sum_{\mathbf{v}\left(P_{b}\right) \in \mathscr{V}(\gamma)}\left(\varrho_{b}-3\right),\left\{\begin{array}{l}
j=0, \ldots, c, \mathbf{V}_{0} \notin \gamma_{0} \\
j=1, \ldots, c, \mathbf{V}_{0} \in \gamma_{0}, \varrho\left(\gamma_{0}\right) \equiv 0 .
\end{array}\right.
\end{aligned}
$$

Then, if $G$ is a nonvacuum graph: $\left(L_{n}(G), L_{\sigma}(G)\right) \neq(0,0)$ and $\hat{G} \notin\{\gamma\}$ or if $G \equiv \Gamma$ is $1 P I$ and $\{\gamma\}$ is such that $\hat{\Gamma} /\{\gamma\}$ contains no fictitious line of $\hat{\Gamma}$, it follows from (2.18) and (2.19) that:

$$
r(\Lambda)+\sum_{j=0}^{c} \max \left\{0, \varrho\left(\gamma_{j}\right)\right\}>0 \quad \text { if } \quad \underline{d}_{Q_{a}} \geqq 3, \quad a=1, \ldots, A(\Lambda) .
$$

When $G$ is a vacuum graph $(\hat{G} \equiv G)$ :

$$
r(\Lambda)+\sum_{j=0}^{c} \max \left\{0, \varrho\left(\gamma_{j}\right)\right\} \geqq 3, \quad \text { if } \quad \underline{d}_{Q_{a}} \geqq 3, \quad a=1, \ldots, A(\Lambda) .
$$

This completes the proof of Theorem 2.2 in the critical theory. We note, that when $\varrho_{a} \equiv \underline{d}_{Q_{a}}, \varrho_{b} \equiv \underline{d}_{P_{b}}, \forall a, b, \varrho(\gamma) \equiv r(\gamma)$.

To prove renormalizability of the theory in the Goldstone phase and the existence of a continuous limit of both phases to the critical theory in the sense of requirement b) of Sect. 1 , the following choice of $\delta(\gamma)$ and $\varrho(\gamma)$ is made in both phases :

$$
\begin{aligned}
& \delta(\gamma)=3-\frac{1}{2}\left(L_{n}(\gamma)-L_{n}^{t p}(\gamma)\right)-2 L_{\sigma}(\gamma)+\sum_{\mathbf{v}\left(Q_{a}\right) \in \mathscr{V}(\gamma)}\left(\delta_{a}-3\right)+\sum_{\mathbf{v}\left(P_{b}\right) \in \mathscr{V}(\gamma)}\left(\delta_{b}-3\right), \\
& \varrho(\gamma)=3-\frac{1}{2}\left(L_{n}(\gamma)-L_{n}^{t p}(\gamma)\right)-2 L_{\sigma}(\gamma)+\sum_{\mathbf{v}\left(Q_{a}\right) \in \mathscr{V}(\gamma)}\left(\varrho_{a}-3\right)+\sum_{\mathbf{V}\left(P_{b}\right) \in \mathscr{V}(\gamma)}\left(\varrho_{b}-3\right),
\end{aligned}
$$

in which $L_{n}^{t p}(\gamma)$ denotes the number of external $n$-lines carrying $n_{F}$-tadpoles (i.e., $\left\langle n_{F}\right\rangle \equiv\langle(n, F)\rangle$-diagrams), $L_{n}^{t p} \neq 0$ only in the Goldstone phase. 
We note, that the choice $(2.20 \mathrm{a})$, when $L_{n}^{t p}(\gamma) \neq 0$ is in agreement with the correct BPHZ renormalization scheme for theories with spontaneous symmetry breaking in which the spontaneous symmetry breaking parameter $\langle n+F\rangle=\left\langle n_{F}\right\rangle+F$ is made soft: $s^{1 / 2}\left(\left\langle n_{F}\right\rangle+F\right)$ to ensure minimal UV subtractions $[34,45,46](n+F$ denotes the unshifted $n$-field operator).

When $L_{n}(\gamma)-L_{n}^{t p}(\gamma)$ is odd the unrenormalized integrand $I_{\gamma}$ contains a factor $s^{1 / 2\left(L_{F}(\gamma)+L_{n}^{t}(\gamma)\right)}$, where $L_{F}(\gamma)+L_{n}^{t p}(\gamma)$ is also odd since $L_{n}(\gamma)+L_{F}(\gamma)$ is always even. In such cases, $\delta(\gamma), \varrho(\gamma)$ in (2.20a) and (2.20b) become formally halfintegers. Then, by definition :

$$
\tau_{\gamma}^{\delta(\gamma), \varrho(\gamma)} S^{1 / 2}(\ldots) \equiv S^{1 / 2} \tau_{\gamma}^{\delta(\gamma)-1 / 2, \varrho(\gamma)-1 / 2} .
$$

By (2.21) factors $s^{1 / 2}$ move to the left of $\tau_{\gamma}$ in the forest formula (2.8) for all $1 P I \gamma$ for which $L_{n}(\gamma)-L_{n}^{t p}(\gamma)$ is odd. Resulting subtraction indices $\delta^{\prime}(\gamma)=\delta(\gamma)-\frac{1}{2}$, $\varrho^{\prime}(\gamma)=\varrho(\gamma)-\frac{1}{2}$ become thus integers. Hence, for such graphs $\gamma$ the IR behaviour of the renormalized integrand is determined by $\varrho^{\prime}(\gamma)$ and the IR criterium (2.13) has to be verified with $\varrho^{\prime}(\gamma)$, instead of $\varrho(\gamma)$. To do this, we observe that the free effective $n_{F}$-propagator between two vertices $\mathbf{V}\left[-\frac{1}{2} \sigma n^{2}\right]^{4}$ is (Fig. 1d):

$$
\begin{aligned}
\mathscr{D}_{F F}^{\text {eff }}\left(p^{2} ; m^{2}(s) ; f^{2} s\right) & =\mathscr{D}\left(p^{2} ; m^{2}(s)\right) \mathscr{S}\left(p^{2} ; m^{2}(s)\right)\left[\mathscr{S}\left(p^{2} ; m^{2}(s)\right)+s f^{2} \mathscr{D}\left(p^{2} ; m^{2}(s)\right)\right]^{-1} \\
\mathscr{D}_{F F}^{\text {eff }}\left(p^{2} ; 0, f^{2}\right) & =\left(p^{2}+16 f^{2}|p|\right)^{-1} .
\end{aligned}
$$

Such lines as well as the corresponding graph will be marked by the superscript "eff". Thus effectively there are four kinds of Lagrangian vertices in the Goldstone phase (Fig. 1e). Now we shall prove that the IR criterium (2.13) is satisfied for each connected graph $G^{\text {eff }}$ (or $1 P I$ graph $\Gamma^{\text {eff }}$ ) when the choice $(2.20 \mathrm{~b}$ ) is made with account for the Definition (2.21). The IR canonical dimension of an effective $n_{F}$-line computed in accordance with the low-momentum behaviour of $\mathscr{D}_{F F}^{\text {eff }}(2.21)$ is $\underline{d}_{n_{F}}^{\text {eff }}=1$. Straightforward computation together with $(2.5)\left(\underline{d}_{n}=\frac{1}{2}, \underline{d}_{\sigma}=\frac{5}{2}\right.$ in the Goldstone phase), gives for $r\left(\Lambda^{\text {eff }}\right)$ :

$$
\begin{aligned}
& r\left(\Lambda^{\text {eff }}\right)=\sum_{\mathcal{Y}_{\mathscr{L}}\left(\Lambda^{\text {eff }}\right)}\left(\underline{d}_{\mathscr{L}}^{\text {eff }}-3\right)+\sum_{\mathbf{v}\left[Q_{a}\right] \in \mathscr{V}\left(\Lambda^{\text {eff }}\right)}\left(\underline{d}_{Q_{a}}^{\text {eff }}-3\right)+\sum_{\mathbf{v}\left[P_{b}\right] \in \mathscr{V}\left(\Lambda^{\text {eff }}\right)} \underline{d}_{P_{b}}^{\text {eff }} \\
& +3\left\{B\left(\gamma_{0}^{\text {eff }}\right)-\left|\mathscr{L}_{B}^{q}\left(\gamma_{0}^{\text {eff }}\right)\right|\right\}+\frac{1}{2}\left[L_{n}\left(\gamma_{0}^{\text {eff }}\right)+L_{n}^{\text {eff }}\left(\gamma_{0}^{\text {eff }}\right)-L_{n}^{t p}\left(\gamma_{0}^{\text {eff }}\right)\right]^{*} \\
& +2 L_{\sigma}\left(\gamma_{0}^{\text {eff }}\right)-\sum_{j=1}^{c}\left\{3-\frac{1}{2}\left[L_{n}\left(\gamma_{j}^{\text {eff }}\right)+L_{n}^{\text {eff }}\left(\gamma_{j}^{\text {eff }}\right)-L_{n}^{t p}\left(\gamma_{j}^{\text {eff }}\right)\right]^{*}\right. \\
& \left.-2 L_{\sigma}\left(\gamma_{j}^{\text {eff }}\right)-3 B\left(\gamma_{j}^{\text {eff }}\right)\right\}+\frac{5}{2}\left\{L_{n}\left(G^{\text {eff }}\right)-\left|\mathscr{L}_{n}^{q}\left(\gamma_{0}^{\text {eff }}\right)\right|\right\} \\
& +2\left\{L_{n}^{\text {eff }}\left(G^{\text {eff }}\right)-\left|\mathscr{L}_{n}^{q, \text { eff }}\left(\gamma_{0}^{\text {eff }}\right)\right|\right\}+\frac{1}{2}\left\{L_{\sigma}\left(G^{\text {eff }}\right)-\left|\mathscr{L}_{\sigma}^{q}\left(\gamma_{0}^{\text {eff }}\right)\right|\right\}+r_{0} \\
& +\frac{1}{2} \sum_{j=1}^{c}\left\{L_{n}^{\text {eff }}\left(\gamma_{j}^{\text {eff }}\right)+L_{n}^{t p}\left(\gamma_{j}^{\text {eff }}\right)+L_{\sigma}\left(\gamma_{j}^{\text {eff }}\right)\right\}-\frac{1}{2} C_{\text {odd }}, \\
& {\left[L_{n}\left(\gamma_{j}^{\text {eff }}\right)+L_{n}^{\text {eff }}\left(\gamma_{j}^{\text {eff }}\right)-L_{n}^{t p}\left(\gamma_{j}^{\text {eff }}\right)\right]^{*}} \\
& = \begin{cases}L_{n}\left(\gamma_{j}^{\text {eff }}\right)+L_{n}^{\text {eff }}\left(\gamma_{j}^{\text {eff }}\right)-L_{n}^{t p}\left(\gamma_{j}^{\text {eff }}\right), & \text { when it is even } \\
L_{n}\left(\gamma_{j}^{\text {eff }}\right)+L_{n}^{\text {eff }}\left(\gamma_{j}^{\text {eff }}\right)-L_{n}^{t p}\left(\gamma_{j}^{\text {eff }}\right)+1, & \text { when it is odd } .\end{cases}
\end{aligned}
$$

4 This is a demonstration of the Goldstone's theorem: no particle corresponds to the $n_{F}$-field 
In $\left(2.18^{\prime}\right) \underline{d}_{\mathscr{L}}^{\text {eff }}, \underline{d}_{Q}^{\text {eff }}, \underline{d}_{P}^{\text {eff }}$ are computed from $(2.4 b)$ with $\underline{d}_{n}^{\text {eff }}$ instead of $\underline{d}_{n}$ for all effective $n_{F}$-lines, $C_{\text {odd }}$ is the number of $\gamma_{j}^{\text {eff }}, j=1, \ldots, c$, for which $L_{n}\left(\gamma_{j}^{\text {eff }}\right)+L_{n}^{\text {eff }}\left(\gamma_{j}^{\text {eff }}\right)-L_{n}^{t p}\left(\gamma_{j}^{\text {eff }}\right)$ is odd, all other notations are the same as in (2.18). Simple analysis shows that if for some $\gamma_{j}^{\text {eff }}$ the corresponding number $L_{n}+L_{n}^{\text {eff }}-L_{n}^{t p}$ is odd, then $L_{j}^{\text {eff }} \geqq 1$ or $L_{n}^{t p} \geqq 1$. Indeed, otherwise all external lines of $\gamma_{j}^{\text {eff }}$ should end in $\left(e_{3}\right)$-vertices (Fig. 1e), therefore $G^{\text {eff }} \equiv \gamma_{j}^{\text {eff }}$, which case is excluded by the conditions of the theorem. Therefore, the last two terms in $\left(2.18^{\prime}\right)$ are always nonnegative. This makes (2.13) obvious for $\underline{d}_{Q_{a}} \geqq 3$.

The requirement $\underline{d}_{Q} \geqq 3$ of Theorem 2.2 could be weakened in the Goldstone phase due to the more favourable (than in the critical theory) low-momentum behaviour of the $\sigma$-propagator:

Corollary 2.3. Euclidean Green's functions (2.7) with an arbitrary number of insertions $Q_{\sigma} \equiv \int d^{3} x \sigma(x)$ exist in each order of $1 / N$ expansion in the Goldstone phase.

Proof. It is necessary to show that the IR criterium (2.13) is satisfied for each $G^{\text {eff }}$ with $\varrho\left(\gamma_{j}^{\text {eff }}\right)$ given by $(2.20 \mathrm{~b})$. This is true, if

$$
\frac{1}{2} \sum_{j=1}^{c}\left\{L_{n}^{\text {eff }}\left(\gamma_{j}^{\text {eff }}\right)\right\}+L_{n}^{t p}\left(\gamma_{j}^{\text {eff }}\right)+L_{\sigma}\left(\gamma_{j}^{\text {eff }}\right)+\sum_{\gamma_{\mathscr{L}}\left(\Lambda^{\text {eff }}\right)}\left(\underline{d}_{\mathscr{L}}^{\text {eff }}-3\right)-\frac{1}{2}\left(C_{\text {odd }}+A\left(\Lambda^{\text {eff }}\right)\right),
$$

where $A\left(\Lambda^{\text {eff }}\right)$ is the number of $Q_{\sigma}$-insertions in $\Lambda^{\text {eff }}$, contributed by the second term in $\left(2.18^{\prime}\right), \underline{d}_{Q_{\sigma}}=\frac{5}{2}, \underline{d}_{\mathscr{L}}^{\text {eff }}=\frac{9}{2}, 4, \frac{7}{2}, 3$ for $\left(e_{1}\right),\left(e_{2}\right),\left(e_{3}\right),\left(e_{4}\right)$ - effective Lagrangian vertices (Fig. 1e). $\sigma$-lines of $Q_{\sigma}$-insertions could be coupled to the vertices of $G^{\text {eff }}$ in the following ways: a) $\sigma$-line $\in L_{\sigma}\left(\gamma_{j}^{\text {eff }}\right)$ for $\gamma_{j}^{\text {eff }} \in\left\{\gamma_{1}, \ldots, \gamma_{c}\right\}$; b) $\sigma$-line $\in \mathscr{L}_{\sigma}\left(\Lambda^{\text {eff }}\right)$ coupled to $\mathscr{V}\left[-\frac{1}{2} \sigma n^{2}\right]$-vertex of $\left(e_{1}\right)$ type; c) $\sigma$-line $\in \mathscr{L}_{\sigma}\left(\Lambda^{\text {eff }}\right)$ coupled to $\left(e_{4}\right)$-vertex which in its turn could be coupled to $\left(e_{3}\right)$-vertex $\in \mathscr{V}_{\mathscr{L}}\left(\gamma_{j}^{\text {eff }}\right)$ (then the corresponding $n_{F}$-line is a tadpole, while in the interesting cases when $L_{n}\left(\gamma_{j}^{\text {eff }}\right)+L_{n}^{\text {eff }}\left(\gamma_{j}^{\text {eff }}\right)-L_{n}^{t p}\left(\gamma_{j}^{\text {eff }}\right)$ is odd at least one of the remaining $L_{n}\left(\gamma_{j}^{\text {eff }}\right)$-lines is effective), or to $\left(e_{3}\right)$-vertex $\in \mathscr{V}_{\mathscr{L}}\left(\Lambda^{\mathrm{eff}}\right)$.

In each case $-\frac{1}{2} A\left(\Lambda^{\text {eff }}\right)$ in (2.23) is canceled by the contribution of $\frac{1}{2} L_{\sigma}\left(\gamma_{j}^{\text {eff }}\right)$, $\underline{d}_{\mathscr{L}}^{\text {eff }}-3$, and $\frac{1}{2} L_{n}^{t p}\left(\gamma_{j}\right)$, leaving thus (2.23) always nonnegative.

Analogous analysis proves:

Corollary 2.4. Euclidean Green's functions with only one insertion of $\int d^{3} x \mathscr{N}_{3}^{3}[Q](x)$, where $Q$ is a monomial in $n^{2}$ and $(n . F)$ with $\underline{d}_{Q} \geqq \frac{3}{2}$ as well as of $\int d^{3} x \mathscr{N}_{3}^{3}\left[(n, F)_{s}^{2}\right](x)$ exist in each order of $1 / N$ expansion in the Goldstone phase.

\section{Quantum Chirality Identities}

This section is devoted to the proof of quantum chirality identities for the soft mass BPHZL - renormalized $1 / N$ expansion of the Green's functions (2.7). They are analogues of the quantum chirality condition in the usual renormalization scheme $[12,24]$ :

$$
\mathscr{N}\left[P(n, \sigma) n^{2}\right](x)=\text { const } \mathscr{N}[P(n, \sigma)](x),
$$


$\mathscr{N}$ denotes Zimmermann's normal product with canonical (minimal) subtractions with "hard" mass.

As we shall see, in the present scheme additional terms appear in the r.h.s. of this relation, due to the more complicated structure of the subtraction operator $\tau^{\delta, \varrho}$.

To formulate quantum chirality identities the concepts of factorized graph and factorization of forests will be necessary (cf. also Zimmermann [30] and Lam [33]).

Definition 3.1. Let $P Q$ be a formal product of two monomials $P$ and $Q$ of the type (2.3). The graph $\Gamma$ containing the composite vertex $\mathrm{V}[P Q]$ is called $P \backslash Q$-factorized if there exist two subgraphs $\gamma^{P}, \gamma^{Q}$ of $\Gamma$ such that $\mathscr{L}\left(\gamma^{P}\right)\left(\mathscr{L}\left(\gamma^{Q}\right)\right)$ contains nonempty set of $P$ - $(Q-)$ lines and no $Q$ - $(P$ - $)$ lines, and $\mathscr{L}\left(\gamma^{P}\right) \cup \mathscr{L}\left(\gamma^{Q}\right)=\mathscr{L}(\Gamma), \mathscr{L}\left(\gamma^{P}\right) \cap \mathscr{L}\left(\gamma^{Q}\right)=\emptyset$, $\mathscr{V}\left(\gamma^{P}\right) \cap \mathscr{V}\left(\gamma^{Q}\right)=\mathbf{V}[P Q]$. Such graph will be denoted $\Gamma \equiv \gamma^{P} \mid \gamma^{Q}$. Let $\tilde{\mathscr{F}}_{\Gamma}$ contain all sets of $\Gamma=\gamma^{P} \mid \gamma^{Q}$-subgraphs (including the empty set $\emptyset$ ).

Definition 3.2. The mapping $\mathfrak{M}: \mathscr{F}_{\Gamma} \rightarrow \tilde{\mathscr{F}}_{\Gamma}$ is called $P \mid Q$-factorization if: i) for all $U \in \mathscr{F}_{\Gamma}, U=\left\{\gamma_{1}, \ldots, \gamma_{c}\right\}, \gamma_{i}$ is not $P \mid Q$ factorized, $i=1, \ldots, c, \mathfrak{M}(U) \equiv U$; ii) for $U=\left\{\gamma_{1}, \ldots, \gamma_{c}, \gamma_{j_{p}}^{P} \mid \gamma_{k_{q}}^{Q}, j_{p}=1, \ldots, L, k_{q}=1,=, M\right\}, \gamma_{i}$ is not $P \mid Q$-factorized, $i=1, \ldots, c$, $\gamma_{i} \neq \gamma_{p}^{P} \neq \gamma_{q}^{Q}, i=1, \ldots, c, p=1, \ldots, L, q=1, \ldots, M ; \gamma_{1}^{P} \neq \gamma_{2}^{P} \neq \ldots \neq \gamma_{L}^{P}, \gamma_{1}^{Q} \neq \gamma_{2}^{Q} \neq \ldots \neq \gamma_{M}^{Q}$, $\mathfrak{M}(U) \equiv\left\{\gamma_{1}, \ldots, \gamma_{c}, \gamma_{p}^{P}, \ldots, \gamma_{L}^{P}, \gamma_{1}^{Q}, \ldots, \gamma_{M}^{Q}\right\} ;$ iii) $\mathfrak{M}(U) \equiv \emptyset$ for all other $U \in \mathscr{F}_{\Gamma}$ (including $U=\emptyset)$.

A special kind of Zimmermann's normal product $\mathscr{N}_{\delta}^{\varrho}[P \mid Q](x)$ is introduced by changing $\mathscr{F}_{\Gamma}$ in the forest formula (2.8) into $\mathfrak{M}\left(\mathscr{F}_{\Gamma}\right)$ for each $P \mid Q$ factorized graph $\Gamma$.

Theorem 3.1. The following chirality identities are true for the Green's functions (2.7) ( $P$ is of form (2.3)) :

$$
\begin{aligned}
& \left\langle\mathscr{N}_{\delta}^{\varrho}\left[P\left(n^{2}+2\left(n, F s^{1 / 2}\right)\right)\right](x) X\right\rangle=\mathscr{D}^{\mathrm{ren}}\left(0 ; m^{2}(s)\right)\left\langle\mathscr{N}_{\delta-1}^{o-1}[P](x) X\right\rangle \\
& +\sum_{a, k} \sum_{r=0}^{k}(-1)^{r}\left(\begin{array}{l}
k \\
r
\end{array}\right) \partial_{(\mu)}^{r}\left\langle\mathscr{N}_{\delta+\delta_{a}-3}^{\varrho+o_{a}-3}\left[\frac{\partial Q_{a}}{\partial\left(\partial_{(\mu)}^{k} \sigma\right)} \mid \partial_{\mu_{r+1}} \ldots \partial_{\mu_{k}} P\right](x) \hat{X}^{Q_{a}}\right\rangle \\
& +2 \sum_{j=1}^{L_{\sigma}} \delta\left(x-x_{j}^{\prime \prime}\right)\left\langle\mathcal{N}_{\delta-1}^{\varrho-1}[P](x) \hat{X}^{\sigma\left(x_{j}^{\prime \prime}\right)}\right\rangle \\
& +\sum_{\{X(1), X(2)\}}\left\langle Y_{\{X(1), X(2)\}}^{\{C(1), C(2)\}}(x) \hat{X}^{C(1) C(2)}\right\rangle,
\end{aligned}
$$

where for simplicity we consider only Green's functions with integrated composite operators :

$$
\begin{aligned}
& X \equiv \prod_{a=1}^{A} Q_{a} X_{n} X_{\sigma}, Q_{a}, X_{n}, X_{\sigma} \text { are the same as in (2.7), } \\
& C(i)=\prod_{a \in A(i)} Q_{a}, A(i) \cong[1, A], i=1,2, A(1) \cap A(2)=\emptyset \\
& \hat{X}^{\sigma(y)} \sigma(y) \equiv \hat{X}^{C(1) C(2)} C(1) C(2) \equiv \hat{X}^{Q} Q \equiv X
\end{aligned}
$$


and $Y_{\{X(1), X(2)\}}^{\{C(1), C(2)\}}(x)$ is the normal product of the monomial:

$$
\begin{aligned}
& \prod_{i=1}^{2} X_{n}^{(i)}(x) X_{\sigma}^{(i)}(x), X_{n}^{(i)}(x) \equiv \prod_{k=1}^{L_{n}^{(i)}} n_{\sigma_{k}}(x), X_{\sigma}^{(i)}(x) \equiv(\sigma(x))^{L_{\sigma}^{(i)}}, \\
& Y_{\{X(1), X(2)\}}^{\{C(1), C(2)\}}(x)=-\mathcal{N}_{\delta(Y)}^{\varrho(Y)}\left[\tau ^ { \delta ( Y ) , \varrho ( Y ) } \left(\left\langle\mathcal{N}_{\delta-1}^{\varrho-1}[P](0) C(1) \tilde{X}_{n}^{(1)} \tilde{X}_{\sigma}^{(1)}\right\rangle^{1 P I}\right.\right. \\
& \left.\left.\cdot\left\langle\sigma(0) C(2) \tilde{X}_{n}^{(2)} \tilde{X}_{\sigma}^{(2)}\right\rangle^{1 P I}\right) X_{n}^{(1)} X_{n}^{(2)} X_{\sigma}^{(1)} X_{\sigma}^{(2)}\right](x), \\
& \varrho(Y)=\varrho+\sum_{a \in A(1) \cup A(2)}\left(\varrho_{a}-3\right), \delta(Y)=\delta+\sum_{a \in A(1) \cup A(2)}\left(\delta_{a}-3\right), \\
& \varrho^{\prime}(Y)=\varrho(Y)-\sum_{i=1}^{2}\left\{\frac{1}{2}\left[L_{n}^{(i)}-L_{n}^{t p(i)}\right]^{*}+2 L_{\sigma}^{(i)}\right\}, \\
& \delta^{\prime}(Y)=\delta(Y)-\sum_{i=1}^{2}\left\{\frac{1}{2}\left[L_{n}^{(i)}-L_{n}^{t p(i)}\right]^{*}+2 L_{\sigma}^{(i)}\right\} .
\end{aligned}
$$

The last sum in (3.1) runs over all possible monomials $\prod_{i=1}^{2} X_{n}^{(i)}(x) X_{\sigma}^{(i)}(x)$ for which one of the inequalities: $\delta^{\prime}(Y) \geqq 0$ and $\varrho^{\prime}(Y) \geqq 1$ is true, " $\sim$ " denotes Fouriertransform. Renormalized self-closed $n$-field loop $\mathscr{D}^{\text {ren }}\left(0 ; m^{2}(s)\right)$ defined in the Appendix (A.3) accounts for the ambiguity in the renormalization ${ }^{5}$.

Proof. To verify (3.1) we analyze all types of graphs contributing to the 1.h.s. of (3.1). They are plotted in Fig. 2. We shall consider only the high-temperature phase and the critical theory (the situation in the low-temperature phase is strictly analogous). The expression corresponding to $\Gamma_{1}$ by (2.8) can be represented as the sum :

$$
R_{\Gamma_{1}}=-\tilde{R}_{\Gamma}+\Lambda_{\Gamma_{1}}^{(1)}+\Lambda_{\Gamma_{1}}^{(2)}+R_{\Gamma_{1}}^{\mathrm{s}} .
$$

Here $\Lambda_{\Gamma_{1}}^{(1)}$ is the contribution of all forests $U \in \mathscr{F}_{\Gamma}$ with at least one element $g \in U$ such that one of the lines $l_{i} \in \mathscr{L}(g), i=1,2$, while the other one does not (Fig. 3a). $\Lambda_{\Gamma_{1}}^{(2)}$ is the contribution of all forests $U \in \mathscr{F}_{\Gamma_{1}}$ with at least one element $\hat{g} \in U$ such that both vertices $\mathbf{V}\left[P n^{2}\right]$ and $\mathbf{v} \in \mathscr{V}(\hat{g})$ and $l_{i} \in \mathscr{L}(\hat{g}), i=1,2,3$ (Fig. $3 \mathrm{~b}$ ). $R_{\Gamma_{1}}^{\mathbf{S}}$ is the contribution of all forests $U \in \mathscr{F}_{\Gamma_{1}}$ with at least one $P \mid Q$-factorized element $\gamma(S)=\gamma^{P} \cup \mathbf{S}, \mathbf{S} \equiv l_{1} \cup l_{2}$ (Fig. 3c). In all remaining forests $U^{\prime} \in \mathscr{F}_{\Gamma}$ any element $\gamma^{\left\{1 P^{2}\right\}} \in U^{\prime}$ such that $\mathbf{v} \in \mathscr{V}\left(\gamma^{\left\{P^{2}\right\}}\right)$ contains the lines $l_{i}, i=1,2,3$ (constituting the graph $\gamma_{0}$ ). In all $U^{\prime}$ the contribution of $\gamma_{0}$ is -1 [cf. Definition (A.6) of the $\sigma$-propagator]. The result is topologically equivalent to $\Gamma$. However, the renormalized expression corresponding to it by $(2.8)$ is :

$$
-\tilde{R}_{\Gamma}=-\sum_{U^{\prime} \in \mathfrak{M}\left(\mathscr{F}_{\Gamma}\right)} \prod_{\gamma \in U}\left(-\tau_{\gamma} I_{\Gamma}\left(U^{\prime}\right)\right) \neq-R_{\Gamma}
$$

Here $\mathfrak{M}$ is a $P \mid n^{2}$-factorization (Definition 3.2).

Analogously, graphs $\Gamma^{\left\{P^{2}, Q\right\}}$ in Fig. $2 b$ give:

$$
R_{\Gamma^{\left\{n^{2}, Q\right\}}}=-\tilde{R}_{\Gamma^{\{P \mid Q\}}}+\Lambda_{\Gamma^{\{}\left\{n^{2}, Q\right\}}^{(1)}+\Lambda_{\Gamma^{\left\{P n^{2}, Q\right\}}}^{(2)} .
$$

5 As a $\gamma^{Q}$-graph [Q-some monomial with $\left.\mu_{n}(Q) \geqq 2\right]$, it is no longer "forbidden". However, the ambiguity, due to its renormalization is not fixed by the normalization conditions for the Green's functions $\left\langle X_{n}\right\rangle(4.12)$ and (4.14), since there it is not allowed 

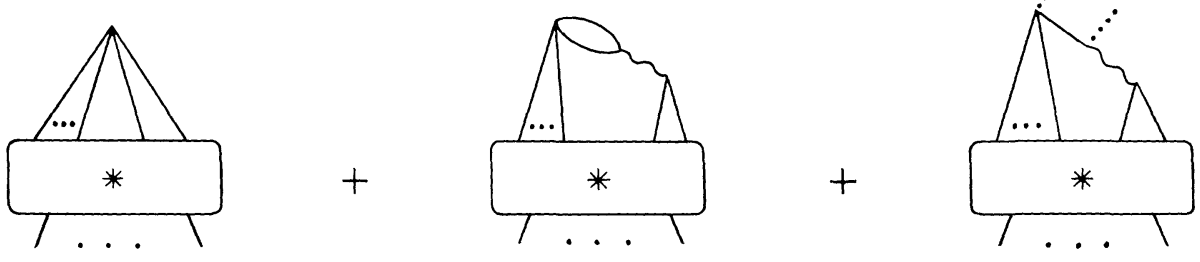

$2 a$
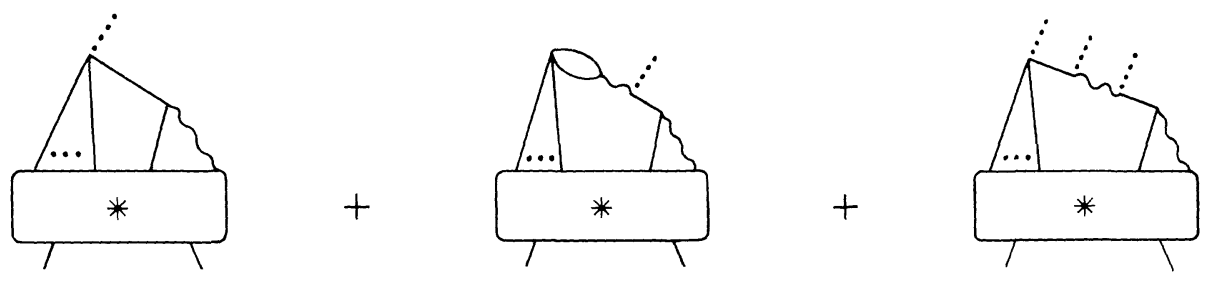

$2 b$
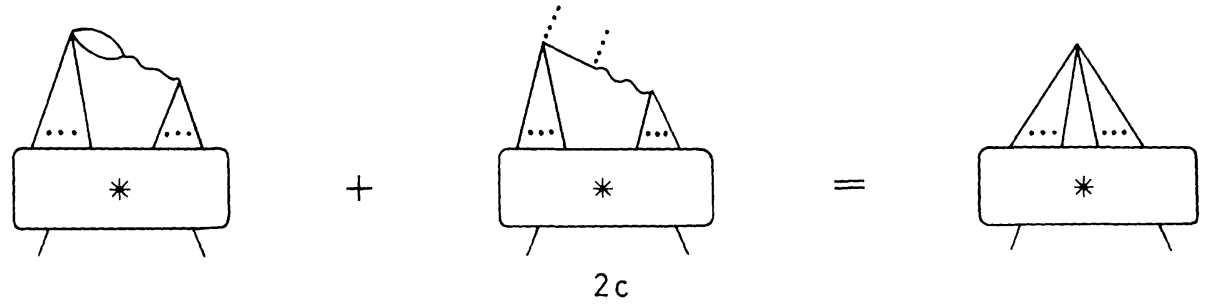

Fig. 2. All possible types of graphs giving rise to chirality identities (3.1)

Lemma 3.2. $\Lambda_{\Gamma_{1}}^{(1)} \equiv \Lambda_{\Gamma^{\left\{P n^{2}, Q\right\}}}^{(1)} \equiv 0$.

Proof. Obviously, $\Gamma_{1}$ is a particular case of $\Gamma^{\left\{\mathrm{Pn}^{2}, Q\right\}}$, which, for shortness, will be denoted $\Gamma^{\{\}}$. Consider the subset $\mathscr{F}_{\Gamma^{\prime}}(g) \subset \mathscr{F}_{\left.\Gamma^{\prime}\right\}}, \mathscr{F}_{\Gamma^{\prime},}(g)=\{U, U$ contains an element $g$ with the property: $g$ is maximal in $U$, for which $\left.l_{1} \notin \mathscr{L}(g), l_{2} \in \mathscr{L}(g)\right\}$

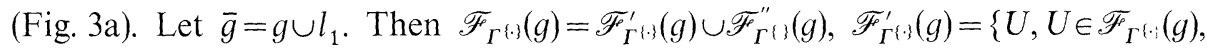
$\bar{g} \notin U\}$. Hence :

$$
\begin{aligned}
\Lambda_{\left.\Gamma^{\{}\right\}}^{(1)}= & S_{\left.\Gamma^{\{}\right\}} \sum_{g} \sum_{U \in \mathscr{F} \Gamma^{(j)}(g)} \prod_{\substack{\lambda \cap \bar{g}=\emptyset \\
\lambda \supset \bar{g}}}\left(-\tau_{\lambda} S_{\lambda} I_{\Gamma^{(j, j} \bar{g}}\right) \\
& \cdot\left\{\left(1-\tau_{\bar{g}}\right) S_{\bar{g}}\left[\mathscr{D}_{l_{1}}\left(-\tau_{g}\left(\bar{R}_{g}\right)\right]\right\}\right.
\end{aligned}
$$

and $\mathscr{D}_{l_{1}}$ is the propagator carried by the line $l_{1}$. From $(2.20)$ follows that $\delta(\bar{g})=\delta(g)+1, \varrho(\bar{g})=\varrho(g)+1$. Since $S_{\bar{g}}\left[\tau_{g} \bar{R}_{g}\right]$ is a polynomial in $S^{g}$ and the external momenta of $\bar{g}\left\{p^{\bar{g}}\right\}$ of order $\leqq \delta(g)$ the expression in the parenthesis is a sum of 


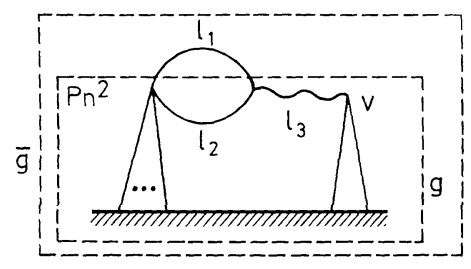

$3 a$

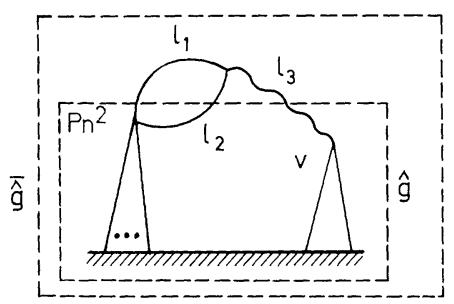

$3 b$

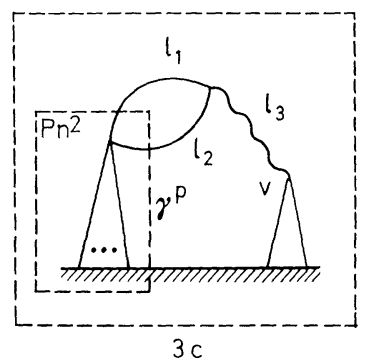

Fig. 3. a Forests contributing to $\Lambda_{\Gamma_{1}}^{(1)}$; b forests contributing to $\Lambda_{\Gamma_{1}}^{(2)} ; \mathbf{c}$ forests contributing to $R^{S}$

terms of the following type $\left(n_{1}+n_{2}+2 k \leqq \delta(g)\right)$ :

$$
\int \frac{d^{3} l}{(2 \pi)^{3}}\left(1-t_{p^{\bar{g}}, s^{\bar{g}}-1}^{\varrho(g)}\right)\left(1-t_{p^{\bar{g}}, s^{\bar{g}}}^{\delta(1)}\right)\left(p^{\bar{g}}\right)^{n_{1}}\left(s^{\bar{g}}\right)^{n_{2}}\left(l^{2}\right)^{k} /\left(m^{2}(s)+l^{2}\right)
$$

or, equivalently:

$$
\begin{aligned}
& \int \frac{d^{3} l}{(2 \pi)^{3}}\left(1-t_{p^{g}, s^{\bar{g}}-1}^{\varrho(\bar{g})}\right)\left(p^{\bar{g}}\right)^{n_{1}}\left(s^{\bar{g}}\right)^{n_{2}}\left(1-\tau_{s^{\bar{g}}}^{\delta(g)+1-n_{1}-n_{2}}\right) \\
& \quad \cdot\left[m^{2 k}(s) /\left(l^{2}+m^{2}(s)\right)+\sum_{r=0}^{k-1}\left(\begin{array}{l}
k \\
r
\end{array}\right) m^{2 r}(s)\left(m^{2}(s)+l^{2}\right)^{k-r-1}\right],
\end{aligned}
$$

where we have used that $\int d^{3} l\left(1-t_{s}^{d}\right)\left[m^{2}(s)+l^{2}\right]^{-1} \equiv 0$ for $d \geqq 1$. This proves Lemma 3.2.

Lemma 3.3. $\Lambda_{\Gamma_{1}}^{(2)}=\Lambda_{\Gamma^{\prime},}^{(2)} \equiv 0$.

The proof completely resembles that of Lemma 3.2. 
Lemma 3.4. Let $\Gamma=\gamma^{P} \mid \gamma^{Q}$ is a $1 P I P \mid Q$-factorized graph. Then the following relation holds:

i) $\bar{R}_{\Gamma}=\bar{R}_{\gamma^{\mathrm{P}}} \bar{R}_{\gamma \mathrm{Q}}+\left(-\tau_{\gamma^{\mathrm{P}}} \bar{R}_{\gamma^{\mathrm{P}}}\right) \bar{R}_{\gamma^{\mathrm{Q}}}+\bar{R}_{\gamma^{\mathrm{P}}}\left(-\tau_{\gamma Q} \bar{R}_{\gamma Q}\right)$

$$
+\sum_{\Gamma_{j} \subset \Gamma, \Gamma_{j}=\gamma_{j}^{P} \mid \gamma_{j}^{Q}}\left[-\tau_{\Gamma_{j}}\left(R_{\gamma^{p}} R_{\gamma_{\rho}}\right)\right] \bar{R}_{\Gamma / \Gamma_{j}}
$$

where the sum is over all nontrivial $1 P I P \mid Q$-factorized subgraphs $\Gamma_{j}$ of $\Gamma$. In the conditions of i) also holds :

ii) $-\tau_{\Gamma} \bar{R}_{\Gamma}=\left(-\tau_{\gamma^{P}} \bar{R}_{\gamma^{P}}\right)\left(-\tau_{\gamma^{Q}} \bar{R}_{\gamma^{Q}}\right)$

$$
-\tau_{\Gamma}\left\{\sum_{\Gamma_{J} \subset \Gamma, \Gamma_{J}=\gamma_{J}^{P} \mid \gamma_{j} Q}\left[-\tau_{\Gamma_{j}}\left(R_{\gamma_{J}^{P}} R_{\gamma_{J} Q}\right)\right] \bar{R}_{\Gamma / \Gamma_{J}}\right\}-\tau_{\Gamma}\left[R_{\gamma^{P}} R_{\gamma Q}\right] .
$$

Proof. It goes by induction. i) and ii) are checked directly when $\Gamma$ is a minimal $P \mid Q$-factorized graph. i) is assumed to be true for all nontrivial $1 P I P \mid Q$-factorized subgraphs $\Gamma_{j}$ of $\Gamma$. Taking $-\tau_{\Gamma_{J}}$ from $\bar{R}_{\Gamma_{j}}$ we obtain all r.h.s. terms in ii) as well as the term:

$$
\left(1-\tau_{\Gamma_{j}}\right) S_{\Gamma_{J}}\left[\left(-\tau_{\gamma_{J}^{\mathrm{P}}} \bar{R}_{\gamma_{J}^{\mathrm{P}}}\right)\left(-\tau_{\gamma_{j} \mathrm{Q}} \bar{R}_{\gamma_{j} \mathrm{Q}}\right)\right]
$$

which vanishes since $\delta\left(\Gamma_{j}\right)=\delta\left(\gamma_{j}^{P}\right)+\delta\left(\gamma^{Q}\right)$.

Now we shall make use of the Bogoliubov's form of the forest formula (2.8) for $R_{\Gamma}$ and $\bar{R}_{\Gamma}[18]$ :

$$
\bar{R}_{\Gamma}=\sum_{\{\lambda\}} I_{\Gamma /\langle\lambda\}} \prod_{j=1}^{\mathrm{c}}\left(-\tau_{\lambda_{j}} R_{\lambda_{j}}\right)
$$

where summation goes over all partitions $\{\lambda\}=\left\{\lambda_{1}, \ldots, \lambda_{c}\right\}$ of $\Gamma$ into sets of nontrivial nonoverlapping $1 P I$ subdiagrams $\lambda_{i}$ of $\Gamma$. These partitions could be of two types: ones which include one $\Gamma_{j}=\gamma_{j}^{P} \mid \gamma_{j}^{Q}-\{\lambda\}^{j}$, and ones which do not: $\{\lambda\}^{0}$. Then:

$$
\begin{aligned}
& \bar{R}_{\Gamma}=\sum_{\left\{\lambda^{0}\right.}\left[I_{\gamma^{P} /\left\{\lambda^{P}\right\}} \prod_{\lambda^{P} \subset \gamma^{P}}\left(-\tau_{\lambda^{P}} \bar{R}_{\lambda^{P}}\right)\right]\left[I_{\gamma Q /\{\lambda Q\}} \prod_{\lambda Q \subset \gamma^{Q}}\left(-\tau_{\lambda Q} \bar{R}_{\lambda Q}\right)\right] \\
& \quad+\sum_{\Gamma_{j} \subset \Gamma} \sum_{\left\{\lambda_{3}{ }^{\prime}\right.} I_{\Gamma /\{\}^{j}} \prod_{\lambda^{P} \subset \gamma^{P}}\left(-\tau_{\lambda^{P}} \bar{R}_{\lambda^{P}}\right)\left(-\tau_{\Gamma_{j}} R_{\Gamma_{j}}\right) \prod_{\lambda^{P} \subset \gamma^{Q}}\left(-\tau_{\lambda Q} \bar{R}_{\lambda Q}\right), \\
& \lambda^{P, Q} \subset \gamma^{P, Q}, \mathbf{V}[P Q] \notin \mathscr{V}\left(\lambda^{P}\right), \mathscr{V}\left(\lambda^{Q}\right) .
\end{aligned}
$$

Accounting the identity: $I_{\Gamma /\{\lambda\}\}} \equiv I_{\left.\gamma^{\mathrm{P}} /\left\{\lambda^{\mathrm{P}}\right\}, \gamma_{j}^{\mathrm{P}}\right\}} . I_{\left.\gamma \boldsymbol{Q}_{/\{\{\lambda Q}, \gamma_{j} Q_{\}}\right\}}$and the inductive assumption ii) for $\Gamma_{j}$ in (3.6) we get:

$$
\begin{aligned}
& \bar{R}_{\Gamma}=\sum_{\left\{\lambda^{P}\right\},\left\{\lambda Q^{\prime}\right\}}\left[I_{\gamma^{P} /\left\{\lambda^{P}\right\}} \prod_{\lambda^{P}}\left(-\tau_{\lambda^{P}} \bar{R}_{\lambda^{P}}\right)\right]\left[I_{\gamma^{Q} /\{\lambda Q\}} \prod_{\lambda Q}\left(-\tau_{\lambda Q} \bar{R}_{\lambda Q}\right)\right] \\
& +\sum_{\Gamma, C \Gamma} \sum_{\{\lambda\}^{j}} I_{\Gamma /\left\{\lambda_{j} j\right.} \prod_{\lambda^{P}}\left(-\tau_{\lambda^{P}} \bar{R}_{\lambda^{P}}\right)\left\{-\tau_{\Gamma_{j}} \sum_{\Gamma_{j}^{k} \subseteq \Gamma_{j}}\left[-\tau_{\Gamma_{j}^{k}}\left(R_{\gamma_{j}^{k}, P} R_{\gamma_{j}^{k}, Q}\right)\right]\right. \\
& \left.\cdot \bar{R}_{\Gamma_{J} / \Gamma_{1}^{k}}\right\} \prod_{\lambda Q}\left(-\tau_{\lambda Q} \bar{R}_{\lambda Q}\right), \Gamma_{j}^{k}=\gamma_{j}^{k, P} \mid \gamma_{j}^{k, Q}, \mathbf{V}[P Q] \in \gamma_{j}^{k, P}, \gamma_{j}^{k, Q} .
\end{aligned}
$$


The first sum in (3.7) gives exactly the first three terms in i), the second sum in (3.7) coincides with the last term in i).

Corollary 3.5. Let $G^{\{P Q\}}$ is any graph containing the composite vertex $\mathbf{V}[P Q]$. Then:

$$
R_{G^{\{P Q\}}}=R_{G^{\{P \mid Q\}}}+\sum_{G^{\{P, Q\}} \Gamma_{\Gamma_{J}}}\left[-\tau_{\Gamma_{j}}\left(R_{\gamma_{j}^{P}} R_{\gamma_{j} Q}\right)\right] \bar{R}_{G^{\{P Q\}} / \Gamma_{j}}, \Gamma_{j} \equiv \gamma_{j}^{P} \mid \gamma_{j}^{Q} .
$$

In (3.8) $R_{G^{\{P Q\}}}$ is calculated by (2.8), while $R_{G^{\{P \mid Q\}}}$ is obtained from (2.8) in which $\mathscr{F}_{G^{\{P Q\}}}$ is changed by $\mathfrak{M}\left(\mathscr{F}_{G^{\{P Q}}\right)$ (Definition 3.2$)$. The sum runs over all $1 P I$ nontrivial $P \mid Q$-factorized subgraphs $\Gamma_{j} \subset G^{\{P Q\}}$.

Application of Lemmas 3.2-3.4 and (3.8) implies the desired quantum chirality relations (3.1).

As a simple illustration of quantum chirality identities (3.1) we give the result for $P=\mathbb{1}$ when there are no composite operators $Q_{a}$, except finite counterterms: $\frac{1}{2} N a \int d^{3} x \sigma(x),-\frac{1}{2} b \int d^{3} x \mathcal{N}_{3}^{3}\left[\left(\partial_{\mu} n\right)^{2}\right]_{\otimes}(x)\{a=0$ in the critical theory and in the Goldstone phase, Sect. 4):

$$
\left\langle\mathscr{N}_{1}^{1}\left[n^{\prime 2}\right](x) X_{n}\right\rangle \equiv 0,\left\langle\mathscr{N}_{1}^{1}\left[n^{\prime 2}\right](0)\right\rangle=\left.N\left[s F^{2}+\mathscr{D}^{\mathrm{ren}}\left(0 ; m^{2}(s)\right)\right]\right|_{s=1}+N a,
$$

$n^{\prime} \equiv n+F$ is the unshifted (at its vacuum expectation value) field operator.

\section{Renormalizability}

In BPHZ framework ambiguity of subtractions (2.8) is accounted for by adding finite counterterms to the effective Lagrangian of the theory (in the sense of Zimmermann [30]). The structure of these counterterms is dictated by the subtraction operators $\tau_{\gamma}$ and the symmetries of the model. All finite counterterms have the form:

$$
\mathscr{Q}_{a} \equiv \int d^{3} x \mathscr{N}_{3}^{3}\left[Q_{a}(n, \sigma)\right]_{\otimes}(x),
$$

where $\left\{Q_{a}(n, \sigma)\right\}$ is the set of all Euclidean and $O(N)$-invariant operators of type (2.3) with canonical UV dimension $\bar{d}_{Q_{a}} \leqq 3$. In the Goldstone phase the requirement for $O(N)$-invariance is modified to a requirement for compatibility with WTIs for spontaneously broken $O(N)$ symmetry (cf. [45]):

$$
\begin{gathered}
0=\int d^{3} x \partial^{\mu}\left\langle\mathscr{I}_{\mu}^{\alpha \beta}(x) \mathscr{N}_{3}^{3}\left[Q_{a}(n, \sigma)\right]_{\otimes}(y) X_{n} X_{\sigma}\right\rangle=-\sum_{i=1}^{L_{n}}\left\langle\mathcal{N}_{3}^{3}\left[Q_{a}(n, \sigma)\right]_{\otimes}(y)\right. \\
\left.\cdot\left\{\left(n_{\alpha}\left(x_{i}^{\prime}\right)+s^{1 / 2} F_{\alpha}\right) \partial / \partial n_{\beta}\left(x_{i}^{\prime}\right)-\left(n_{\beta}\left(x_{i}^{\prime}\right)+s^{1 / 2} F_{\beta}\right) \partial / \partial n_{\alpha}\left(x_{i}^{\prime}\right)\right\} X_{n} X_{\sigma}\right\rangle, \\
\mathscr{I}_{\mu}^{\alpha \beta}(x)=(1+b)\left\{\mathscr{N}_{2}^{2}\left[n_{\alpha}\left(\partial_{\mu} n_{\beta}\right)-\left(\partial_{\mu} n_{\alpha}\right) n_{\beta}\right](x)+s^{1 / 2}\left(F_{\alpha} \partial_{\mu} n_{\beta}(x)-F_{\beta} \partial_{\mu} n_{\alpha}(x)\right)\right\} .
\end{gathered}
$$

Here $b$ is taken equal to the coefficient of the field-renormalization counterterm (4.4) to assure correct normalization of the current $\mathscr{I}_{\mu}^{\alpha \beta}$. To check (4.2) quantum 
equations of motion should be used [33]:

$$
\begin{aligned}
& \left\langle\mathscr{N}_{\delta}^{\varrho}\left[P(n, \sigma) \mid \frac{\partial \mathscr{L}_{\text {eff }}}{\partial n_{\alpha}}-\partial_{\mu} \frac{\partial \mathscr{L}_{\text {eff }}}{\partial\left(\partial_{\mu} n_{\alpha}\right)}\right](x) X\right\rangle=-\sum_{a}\left\{\sum_{k_{a}} \sum_{r=0}^{k_{a}}(-1)^{r}\left(\begin{array}{c}
k_{a} \\
r
\end{array}\right)\right\} \\
& \cdot \partial_{(\mu)}^{r} \delta\left(x-x^{a}\right)\left\langle\mathscr{N}_{\delta+\delta_{a}-r-3}^{\underline{a}+Q_{a}-r-3}\left[\frac{\partial P_{a}}{\partial\left(\partial_{(\mu)}^{k_{a}} n_{\alpha}\right)} \mid \partial_{\mu_{r+1}} \ldots \partial_{\mu_{k a}} P(n, \sigma)\right]\left(r_{a}\right) \hat{X}^{\mathfrak{H}_{\dot{\delta}_{a}}^{\rho_{a}}\left[P_{a}\right]\left(x_{a}\right)}\right\rangle \\
& -\sum_{l=1}^{L_{n}} \delta\left(x-x_{l}^{\prime}\right)\left\langle\mathcal{N}_{\delta-5 / 2}^{\varrho-5 / 2}[P(n, \sigma)](x) \hat{X}^{n_{\alpha}\left(x^{i}\right)}\right\rangle, \\
& X \equiv \hat{X}^{\mathscr{N} \varrho_{\delta_{a}}\left[P_{a}\right]\left(x_{a}\right)} \mathcal{N}_{\delta_{a}}^{\varrho_{a}}\left[P_{a}\right]\left(x_{a}\right) \equiv \hat{X}^{n_{\alpha}\left(x_{i}\right)} n_{\alpha}\left(x_{l}^{\prime}\right) \equiv \prod_{a} \mathcal{N}_{\delta_{a}}^{\varrho_{a}}\left[P_{a}\right]\left(x_{a}\right) X_{n} X_{\sigma}, \\
& \mathscr{L}_{\text {eff }}\left(\left\{c_{a}\right\} ; x\right)=-\frac{1}{2} \mathcal{N}_{3}^{3}\left[\left(\partial_{\mu} n\right)^{2}\right]_{\otimes}(x)-N / 2 \mathcal{N}_{3}^{3}\left[\sigma\left(\tilde{\mathscr{G}}^{-1} \sigma\right)\right]_{\otimes}(x)-\frac{1}{2} \mathcal{N}_{3}^{3}\left[m^{2}(s) n^{2}\right]_{\otimes}^{*}(x) \\
& \frac{1}{2} \mathcal{N}_{3}^{3}\left[\sigma\left(n^{2}+2\left(n, F s^{1 / 2}\right)\right)\right]_{\otimes}^{*}(x)+\sum_{a} C_{a} \mathscr{N}_{3}^{3}\left[Q_{a}(n, \sigma)\right]_{\otimes}(x),
\end{aligned}
$$

where "p" in (4.3) indicates that the normal product is factorized (Definition 3.2), $\tilde{\mathscr{G}}$ in (4.4) is the Fourier transform of the $\sigma$-field propagator (A.5) and " $*$ " reminds that "forbidden" graphs (Fig. 1) are omitted in the corresponding normal product. (Note, that there is no "*" in the counterterms $\mathscr{N}_{3}^{3}\left[Q_{a}(n, \sigma)\right]_{\otimes}$.)

The requirement for $O(N)$-compatibility of $Q_{a}$, together with (4.3) and (4.4), gives:

$$
\left[\left(n_{\alpha}(x)+s^{1 / 2} F_{\alpha}\right) \frac{\partial}{\partial n_{\beta}(x)}-\left(n_{\beta}(x)+s^{1 / 2} F_{\beta}\right) \frac{\partial}{\partial n_{\alpha}(x)}\right] Q_{a}(n, \sigma) \equiv 0 .
$$

Hence, all $Q_{a}(n, \sigma)$ in (4.1) depend only on $\left(n+s^{1 / 2} F\right)^{2}$ and $\sigma$ and can be obtained from the corresponding counterterms in the $O(N)$-symmetric phase by translation: $n_{\alpha} \rightarrow n_{\alpha}+s^{1 / 2} F_{\alpha}$ (cf. [23]). Therefore, we shall restrict ourself to the of the high-temperature phase and the critical theory.

All possible counterterms $Q_{a}(n, \sigma)$ in the high-temperature phase have the form:

$$
\begin{array}{llr}
Q_{1}(n)=-\frac{1}{2} n^{2}, & Q_{2}(n)=\frac{1}{4}\left(n^{2}\right)^{2}, & Q_{3}(n)=\frac{1}{6}\left(n^{2}\right)^{3}, \\
Q_{4}(n)=-\frac{1}{2}(1-s) n^{2}, & Q_{5}(n)=-\frac{1}{2}(1-s)^{2} n^{2}, & Q_{6}(n)=\frac{1}{4}(1-s)\left(n^{2}\right)^{2}, \\
Q_{7}(\sigma)=N / 2 \sigma, & Q_{8}(n)=-\frac{1}{2}\left(\partial_{\mu} n\right)^{2}, & Q_{9}(n, \sigma)=-\frac{1}{2} \sigma n^{2} .
\end{array}
$$

However, not all of them are linearly independent ${ }^{6}$ :

6 Linear independence between composite operators $\mathscr{N}\left[Q_{l}(n, \sigma)\right](x)$ will be understood in the weak sense:

$$
\sum_{i=1}^{M} \alpha_{i}\left\langle\mathscr{N}\left[Q_{i}(n, \sigma)\right](x) X_{n}\right\rangle \equiv 0
$$

only for $\alpha_{i}=0, i=1, \ldots, M$ 
Theorem 4.1. In the set of all Euclidean and $O(N)$-invariant (compatible with WTIs for spontaneously broken $O(N)$-symmetry in the Goldstone phase) composite operators $\mathscr{N}_{\delta}^{\varrho}[Q(n, \sigma)]_{\otimes}(x)$ with $\delta, \varrho \leqq 3$, there are only two which are linearly independent: $\sigma(x)$ and $\mathscr{N}_{3}^{3}\left[\left(\partial_{\mu} n\right)^{2}\right]_{\otimes}(x)$.

For the remaining two independent counterterms (4.1) the following notation is introduced:

$$
\Delta_{0} \equiv N / 2 \int d^{3} x \sigma(x), \quad \Delta_{1} \equiv-\frac{1}{2} \int d^{3} x \mathscr{N}_{3}^{3}\left[\left(\partial_{\mu} n\right)^{2}\right]_{\otimes}(x) .
$$

Proof. It relies on the two types of linear relations in the formal algebra of composite operators: (a) algebraic ZIs. [31] and: (b) quantum chirality identities (Theorem 3.1).

ZIs for $\mathscr{N}_{3}^{3}\left[Q_{a}\right]_{\otimes}(x), a=4,5,6(4.5)$ are derived with the help of formulas (cf. $[25,42])\left(D_{p, s}^{d} \equiv t_{p, s}^{d}-t_{p, s}^{d-1}, p \equiv p^{\gamma}, s \equiv s^{\gamma}, \delta \equiv \delta(\gamma), \varrho \equiv \varrho(\gamma)\right)$ :

$$
\begin{aligned}
\tau_{\gamma}^{\delta, \varrho}(1-s)^{k}= & (1-s)^{k} \tau_{\gamma}^{\delta, \varrho}, \quad \gamma \nexists \mathbf{V}\left[Q_{a}\right], \quad k=1,2, \quad a=4,5,6, \\
\tau_{\gamma}^{\delta, \varrho}(1-s)= & (1-s) \tau_{\gamma}^{\delta-1, \varrho-1}+\left[\left(1-t_{p, s-1}^{\varrho-1}\right) D_{p, s}^{\delta}\right]\left(1-\tau_{\gamma}^{\delta-1, \varrho-1}\right), \quad \gamma \ni \mathbf{V}\left[Q_{a}\right], \\
\tau_{\gamma}^{\delta, \varrho}(1-s)^{2}= & (1-s)^{2} \tau_{\gamma}^{\delta-2, \varrho-2}+\left[\left(1-t_{p, s-1}^{\varrho-1}\right)\left(D_{p, s}^{\delta}-D_{p, s}^{\delta-1}\right)\right. \\
& \left.+2(1-s)\left(1-t_{p, s-1}^{\varrho-2}\right) D_{p, s}^{\delta-1}\right]\left(1-\tau_{\gamma}^{\delta-2, \varrho-2}\right), \quad \gamma \ni \mathbf{V}\left[Q_{a}\right] .
\end{aligned}
$$

Straightforward computation gives:

$$
\left\langle\mathcal{N}_{3}^{3}\left[Q_{a}(n, \sigma)\right]_{\otimes}(x) X_{n}\right\rangle=\sum_{\substack{i=3 \\ i \neq 7}}^{9} \varrho_{a, i}(m / \mu)\left\langle\mathcal{N}_{3}^{3}\left[Q_{i}(n, \sigma)\right]_{\otimes}(x) X_{n}\right\rangle,
$$

where the coefficient functions $\varrho_{a, i}(m / \mu), a=4,5,6$ are explicitely expressed in terms of vertex $(1 P I)$ Green's functions.

ZIs for $Q_{1}, Q_{2}$ are obtained with the help of the identity:

$$
\begin{aligned}
& \tau_{\gamma}^{\delta, \varrho}=\tau_{\gamma}^{\delta-k, \varrho-k}+\tau_{\gamma}^{\delta, \varrho}\left(1-\tau_{\gamma}^{\delta-k, \varrho-k}\right), \quad \gamma \ni \mathbf{V}\left[Q_{i}\right], \quad k, i=1,2, \\
& \left\langle\mathscr{N}_{3}^{3}\left[n^{2}\right]_{\otimes}(x) X_{n}\right\rangle-\sum_{a=1}^{9} \bar{\varrho}_{1, a}(m / \mu)\left\langle\mathscr{N}_{3}^{3}\left[Q_{a}(n, \sigma)\right]_{\otimes}(x) X_{n}\right\rangle \\
& \quad=\left\langle\mathscr{N}_{1}^{1}\left[n^{2}\right]_{\otimes}(x) X_{n}\right\rangle=0, \\
& \left\langle\mathcal{N}_{3}^{3}\left[\left(n^{2}\right)^{2}\right]_{\otimes}(x) X_{n}\right\rangle-\left\langle\mathscr{N}_{2}^{2}\left[\left(n^{2}\right)^{2}\right]_{\otimes}(x) X_{n}\right\rangle \\
& \quad=\sum_{a=1}^{9} \bar{\varrho}_{2 . a}(m / \mu)\left\langle\mathscr{N}_{3}^{3}\left[Q_{a}(n, \sigma)\right]_{\otimes}(x) X_{n}\right\rangle .
\end{aligned}
$$

Here coefficient functions $\bar{\varrho}_{i, a}(m / \mu), i=1,2, a=1, \ldots, 9$ are again expressed in terms of $1 P I$ Green's functions. The first Eq. (4.9) vanishes due to the chirality identity (3.9).

Now we shall make use of $(3.1)$ for $L_{\sigma}=0$ and $P=\mathscr{N}_{3}^{3}\left[Q_{3}\right]_{\otimes}(x), \mathscr{N}_{3}^{3}\left[Q_{9}\right]_{\otimes}(x)$ and $\mathscr{N}_{2}^{2}\left[\left(\mathrm{n}^{2}\right)^{2}\right]_{\otimes}(\mathrm{x})$, respectively. Note, that for all of them the first term in r.h.s. of (3.1) is not present, since responsible for it $\mathscr{D}^{\text {ren }}\left(0 ; m^{2}(s)\right)$ arises in counterterms as $\left(1-\tau^{1,1}\right) \mathscr{D}\left(0 ; m^{2}(s)\right) \equiv 0$, because of (A.3) (counterterms are in so called in- 
termediate normalization [34]). The result reads :

$$
\begin{aligned}
& \left\langle\mathscr{N}_{3}^{3}\left[Q_{3}\right]_{\otimes}(x) X_{n}\right\rangle=\sum_{a=1}^{9} d_{3, a}(m / \mu)\left\langle\mathscr{N}_{3}^{3}\left[Q_{a}\right]_{\otimes} X_{n}\right\rangle, \\
& \left\langle\mathscr{N}_{3}^{3}\left[Q_{9}\right]_{\otimes}(x) X_{n}\right\rangle=N C_{7}\left\langle\sigma(x) X_{n}\right\rangle .
\end{aligned}
$$

Chirality identity (3.1) for $\mathcal{N}_{2}^{2}\left[\left(n^{2}\right)^{2}\right]_{\otimes}(x)$ gives rise to a new normal product $\mathscr{N}_{2}^{2}\left[(1-s) n^{2}\right]_{\otimes}(x)$, which is readily expressed with the help of $(4.7)$ in terms of $\mathscr{N}_{2}^{2}\left[\left(n^{2}\right)^{2}\right]_{\otimes}(x)$ and $\sigma(x)$. Finally

$$
\left\langle\mathscr{N}_{2}^{2}\left[\left(n^{2}\right)^{2}\right]_{\otimes}(x) X_{n}\right\rangle=d_{2,7}(m / \mu)\left\langle\sigma(x) X_{n}\right\rangle .
$$

Coefficients functions in $(4.10 \mathrm{~b})$ and $(4.10 \mathrm{c})$ are also given in terms of vertex functions.

The linear set of Eqs. (4.9) and (1.10a)-(4.10c) has a nondegenerate determinant (at least in each order on $1 / N)$ and, therefore, all $\mathscr{N}_{3}^{3}\left[Q_{a}(n, \sigma)\right]_{\otimes}(x), a=1, \ldots, 9$, and $\mathscr{N}_{2}^{2}\left[\left(n^{2}\right)^{2}\right]_{\otimes}(x)$ are expressed as linear combinations of only two of them $\mathscr{Z}_{7,8}$.

The proof goes along the same lines in the critical theory and in the Goldstone phase and is omitted.

Corollary 4.2. The theory in both phases as well as at the critical point is renormalizable. The only independent counterterms will be denoted:

a) In the high-temperature phase: $a(m, \mu) \Delta_{0} \equiv m \tilde{a}(m / \mu) \Delta_{0}$ and $b(m / \mu) \Delta_{1}, \Delta_{0}$ and $\Delta_{1}$ are from (4.6);

(b) low-temperature phase: $a\left(f^{2}, \mu\right) \Delta_{0} \equiv \mu \tilde{a}\left(f^{2} / \mu\right) \Delta_{0}$ and $b\left(f^{2} / \mu\right) \Delta_{1}$;

c) in the critical theory: $b(0) \Delta_{1}$.

Thus the effective Lagrangian (4.4) acquires the form:

$$
\begin{aligned}
\mathscr{L}_{\text {eff }}(x ; m, f, \mu)= & -(1+b) / 2 \mathscr{N}_{3}^{3}\left[\left(\partial_{\mu} n\right)^{2}\right]_{\otimes}(x)+N / 2 \mathscr{N}_{3}^{3}\left[\sigma\left(\tilde{\mathscr{G}}^{-1} \sigma\right)\right]_{\otimes}(x) \\
& -1 / 2 \mathscr{N}_{3}^{3}\left[m^{2}(s) n^{2}\right]_{\otimes}^{*}(x)-1 / 2 \mathscr{N}_{3}^{3}\left[\sigma\left(n^{2}+2\left(n, F s^{1 / 2}\right)\right)\right]_{\otimes}^{*} \\
& +N a / 2 \sigma(x) .
\end{aligned}
$$

The last term in $\left(4.4^{\prime}\right)$ is absent in case c).

Remark 1. The requirement for IR finiteness in the critical theory (Theorem 2.2) restricts all possible counterterms (4.5) only to those with $\underline{d}_{Q_{a}}=3$, whereby the masslessness of the theory is assured (otherwise, resummation of $a(\mu) \Delta_{0^{-}}$ counterterm insertions will lead to a massive theory, cf. Sect.7). The only remaining counterterm is $b(0) \Delta_{1}$.

Remark 2. For the sake of rigour in the Goldstone phase, one should add and substract in $\mathscr{L}_{\text {eff }}\left(4.4^{\prime}\right)$ the term $\left(m_{0}\right.$ is arbitrary mass scale parameter):

$$
\mp \mathscr{N}_{3}^{3}\left[\left(m_{0}^{2}+2 m_{0}\left(\mu-m_{0}\right)(1-s)-m_{0}\left(2 \mu-m_{0}\right)(1-s)^{2} n^{2}\right]_{\otimes}^{*}(x) .\right.
$$

The minis term (4.11), together with the term $-1 / 2 \mathscr{N}^{3}\left[(1-s)^{2} \mu^{2} n^{2}\right]_{\odot}^{*}(x)$ gives a mass $m_{0}(s)=m_{0} s+(1-s) \mu$ to the $n$ - and $\sigma$-propagators (A.4) and (A.5) and the remainder in (4.11) is considered as an insertion in the Green's functions, which modifies the coefficients of the counterterms. After expressing the latter as linear combinations of $\Delta_{0}$ and $\Delta_{1}$ according to Theorem $4.1, m_{0}$ is safely set equal to zero. 

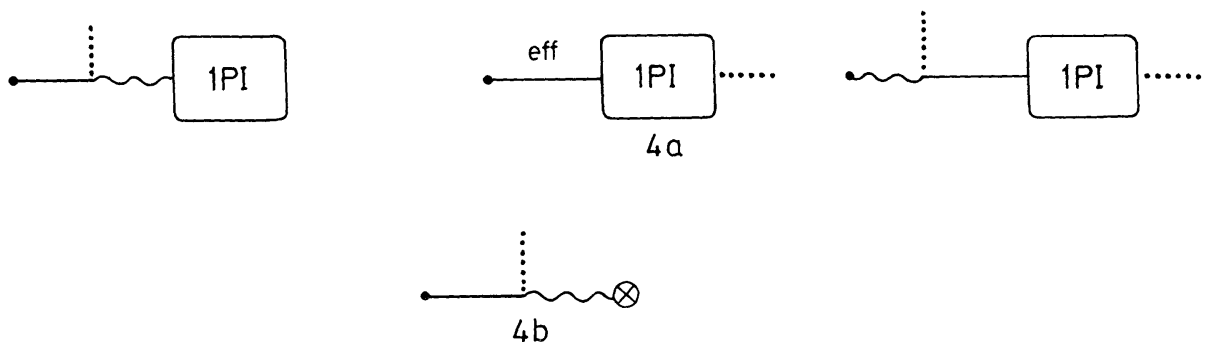

Fig. 4. Possible (sub-)graphs for $\left\langle n_{F}\right\rangle$

To fix the two finite counterterms in the high-temperature phase physical normalization conditions are imposed:

$$
\left.\Gamma^{(2,0)}\left(p^{2} ; m, \mu\right)\right|_{p^{2}=-m^{2}}=0,\left.\quad \Gamma^{(2,0)}\left(p^{2} ; m, \mu\right)\right|_{p^{2}=\mu^{2}}=-\left(\mu^{2}+m^{2}\right),
$$

where $\Gamma^{(2,0)}$ is the $O(N)$-invariant part of the two point vertex function of the $n$ field $\left(\Gamma^{(2,0)}\right.$ is $1 P I$ with respect only of the $n$-lines). Counterms $a(m, \mu)$ and $b(m / \mu)$ are determined by (4.12) recursively in each order $k$ of $1 / N$ by:

$$
\begin{aligned}
& 8 \pi m^{2} \tilde{a}^{(k)}(m / \mu)=-m^{2} /\left.\left(m^{2}+\mu^{2}\right) \Pi^{(k)}\left(p^{2} ; m, \mu\right)\right|_{p^{2}=\mu^{2}}-\mu^{2} /\left(m^{2}+\mu^{2}\right) \\
& \left.\quad \Pi^{(k)}\left(p^{2} ; m, \mu\right)\right|_{p^{2}=-m^{2}}, \\
& b^{(k)}(m / \mu)=\left(m^{2}+\mu^{2}\right)^{-1}\left[\left.\Pi^{(k)}\left(p^{2} ; m, \mu\right)\right|_{p^{2}=\mu^{2}}-\left.\Pi^{(k)}\left(p^{2} ; m, \mu\right)\right|_{p^{2}=-m^{2}}\right] .
\end{aligned}
$$

Here $\Pi \equiv \Gamma^{(2,0)}+(1+b) p^{2}$ is the contribution of all nontree graphs of $\Gamma^{(2,0)}, \Pi^{(k)}$ is its $k$-th order in $1 / N$, eventually containing insertions of $a^{(l)} \Delta_{0}$ and $b^{(l)} \Delta_{1}$, of order $l \leqq k-1$.

Physical normalization conditions in the Goldstone phase read:

$$
\left\langle n_{F}\right\rangle=0,\left.\quad \Gamma_{\perp}^{(2,0)}\left(p^{2} ; f^{2}, \mu\right)\right|_{p^{2}=\mu}=-\mu^{2},
$$

the lower index " $\perp$ " denoting projection along isodirections orthogonal to $F$. The first condition (4.14) implies: $\tilde{a}\left(f^{2} / \mu\right)=0$. Indeed, a tadpole $\left\langle n_{F}\right\rangle$-graph with no $a \Delta_{0}$-insertions could possibly contain as subgraphs the graphs plotted in Fig. $4 a$. However, the analysis of the behaviour at zero momentum in the limit $s \rightarrow 1$ of the external $\mathscr{G}_{-}, \mathscr{D}_{F F^{-}}^{\text {eff }}$, and $\mathscr{D}_{F F^{-}}$-propagators and the attached to them $1 P I$ coefficient functions (determined by the subtraction operators $\left(1-\tau^{1,1}\right)$, and $\left(1-\tau^{2,2}\right)$, respectively) shows that the latter vanish. The counterterm $a \Delta_{0}$ contributes to $\left\langle n_{F}\right\rangle$-graphs only in the form of the subgraph in Fig. 4 b.

The second counterterm $b\left(f^{2} / \mu\right)$ can be expressed in terms of nontree contributions $\Pi_{\perp}$ to $\Gamma^{(2,0)}$ in each order $k$ of $1 / N$ :

$$
b^{(k)}\left(f^{2} / \mu\right)=\left.\mu^{-2} \Pi_{\perp}\left(p^{2} ; f^{2}, \mu\right)\right|_{p^{2}=\mu^{2}} .
$$

The single counterterm $b(0)$ in the critical theory (Remark 1 ) is fixed by the normalization condition:

$$
\left.\Gamma^{(2,0)}\left(p^{2} ; \mu\right)\right|_{p^{2}=\mu^{2}}=-\mu^{2}, \quad b^{(k)}(0)=\left.\mu^{-2} \Pi^{(k)}\left(p^{2} ; \mu\right)\right|_{p^{2}=\mu^{2}} .
$$

The critical theory is conformally invariant (after summing up all orders of $1 / N)$. This was proved in [40] by means of explicitely constructed "improved" 
quantum conserved stress-energy tensor:

$$
\Theta_{\mu \nu}(x)=(1+b(0)) \mathscr{N}_{3}^{3}\left[\left(\partial_{\mu} n, \partial_{\nu} n\right)\right](x)-1 / 2 \delta_{\mu \nu}\left(1+b(0)+r_{1}\right) \mathscr{N}_{3}^{3}\left[\left(\partial_{\lambda} n\right)^{2}\right](x),
$$

where the constant $r_{1}$ arises in the $\mathrm{ZI}$ :

$$
\mathscr{N}_{3}^{3}\left[\mu^{2}(1-s)^{2} n^{2}\right](x)=r_{1} \mathscr{N}_{3}^{3}\left[\left(\partial_{\lambda} n\right)^{2}\right](x)
$$

in the critical theory.

Remark 3. In the case $L_{\sigma} \neq 0$ in the Green's functions (2.7), chirality identity (4.10a) is modified, due to the nonvanishing contribution of the contact terms (third sum) in (3.1), and reads:

$$
\left\langle\mathscr{N}_{3}^{3}\left[\sigma n^{2}\right]_{\otimes}(x) X_{n} X_{\sigma}\right\rangle=N C_{7}\left\langle\sigma(x) X_{n} X_{\sigma}\right\rangle+2 \sum_{j=1}^{L_{\sigma}} \delta\left(x-x_{j}^{\prime \prime}\right)\left\langle X_{n} X_{\sigma}\right\rangle .
$$

Hence, an additional finite counterterm $-1 / 2 C \int \mathcal{N}_{3}^{3}\left[\sigma n^{2}\right]_{\otimes}(x) d^{3} x \equiv C \Delta_{2}$ arises in the Green's functions $\left\langle X_{n} X_{\sigma}\right\rangle,\left(L_{n}, L_{\sigma}\right) \neq(0,0)$. It can be fixed by imposing normalization condition, analogous to the one in the noncanonically renormalized $O(N)$-invariant $\left(\varphi^{2}\right)_{3}^{2}$ model $[39,40]$ :

$$
\left.\left.\Gamma_{\alpha \beta}^{(2,1)}\left(p_{1}, p_{2} ; m, \mu\right)\right|_{s . p . \mu^{2}} \equiv\left\langle\sigma(0) \tilde{n}_{\alpha}\left(p_{1}\right) \tilde{n}_{\beta}\left(p_{2}\right)\right\rangle^{1 P I}\right|_{p_{1}^{2}=p_{2}^{2}=p_{1}, p_{2}=\mu^{2}}=-\delta_{\alpha \beta}
$$

in the high-temperature phase and the critical theory, and in the Goldstone phase:

$$
\left.\left.\Gamma_{\perp}^{(2,1)}\left(p_{1}, p_{2} ; m, \mu\right)\right|_{s . p \cdot \mu^{2}} \equiv\left\langle\sigma(0) \tilde{n}_{\alpha}\left(p_{1}\right) \tilde{n}_{\beta}\left(p_{2}\right)\right\rangle^{1 P I}\right|_{p_{1}^{2}=p_{2}^{2}=p_{1}, p_{2}=\mu^{2}}=-\delta_{\alpha \beta}^{\perp},
$$

where " $\perp$ " again denotes projection along isodirections orthogonal to $F$.

\section{Critical Limit of the High and Low-Temperature Phases}

The physical normalization conditions (4.12) and (4.14) lead to reparametrization of the theory in both phases : instead of the initial parameter - the "temperature" $T$, the Green's functions of the chiral field depend on $m$ (the physical mass), respectively on $f$ [the physical (spontaneous) magnetization] [23]. Therefore, we have to verify that the limits $m \rightarrow 0$ and $f \rightarrow 0$ in all orders of the renormalized $1 / N$ expansion correspond to the critical limits $T \rightarrow T_{c}+0$ and $T \rightarrow T_{c}-0$, respectively, i.e., to the limit to the renormalized theory at the critical point, which is constructed independently by means of the $1 / N$ diagram technique with $m=f=0$ [cf. (A.4) and (A.5)].

First we consider the high-temperature phase. It is clear from Theorems 2.1 and 2.2, that each renormalized diagram, without finite counterterm insertions $a \Delta_{0}, b \Delta_{1}$, has a well defined limit when $m \rightarrow 0$ to the corresponding diagram of the critical theory $(m \equiv 0)$. To analyze the remaining graphs we shall use the following:

Lemma 5.1. $\tilde{a}^{(k)}(m / \mu)=O\left((\ln m / \mu)^{z}\right), \lim _{m \rightarrow 0} b^{(k)}(m / \mu)=b^{(k)}(0)$,

where the latter coincides with $(4.16)^{7}$.

7 Here and in what follows $z$ will denote powers of logarithms, whose explicit values are not essential for the present discussion 
The proof proceedes by induction in orders of $1 / N$ with the aid of the formulas (4.13). Assume that the lemma holds up to order $k-1$. Then the first term in (4.13a) and $(4.13 b) \Pi^{(k)}\left(\mu^{2} ; m, \mu\right)$ contains only $b^{\left(k^{\prime}\right)}(m / \mu), \tilde{a}^{\left(k^{\prime}\right)}(m / \mu)$ with $k^{\prime} \leqq k-1$ and thus it converges continuously to the corresponding one of the critical theory. Indeed, we only need to show that all graphs of $\Pi^{(k)}\left(\mu^{2} ; m, \mu\right)$ with at least one $m \tilde{a}(m / \mu) \Delta_{0^{-}}$insertion vanish in the limit $m \rightarrow 0$. The latter graphs are of the form ${ }^{8}$.

$$
m^{2 l_{\sigma}} \prod_{j=1}^{l_{\sigma}} \tilde{a}^{\left(k_{j}\right)}(m / \mu) \mathscr{R}_{\Gamma_{\left(2, l_{\sigma}\right)}}\left(\mu^{2} ; 0, \ldots, 0 ; m, \mu\right), \sum_{j=1}^{l_{\sigma}} k_{j}<k-1 .
$$

Applying to (5.1) Weinberg's power counting theorem [47] about asymptotic behaviour of diagrams in the momenta and/or other dimensional parameters (generalized to the case of the soft mass BPHZL renormalization scheme in the Appendix of [40]) and accounting for the inductive assumption, we have:

$$
\begin{gathered}
\varepsilon^{2 l_{\sigma}} m^{2 l_{\sigma}} \prod_{j=1}^{l_{\sigma}} \tilde{a}^{\left(k_{J}\right)}(\varepsilon m / \mu) \mathscr{R}_{\Gamma_{\left(2, l_{\sigma}\right)}}\left(\mu^{2} ; 0, \ldots, 0 ; \varepsilon m, \mu\right) \underset{\varepsilon \rightarrow 0}{\approx_{0}} \operatorname{const} \varepsilon^{2}(\ln \varepsilon)^{z} \\
\cdot \mathscr{R}_{\Gamma_{\left(2, l_{\sigma}\right)}}\left(\mu^{2} / \varepsilon^{2} ; 0, \ldots, 0 ; m, \mu / \varepsilon\right) \underset{\varepsilon \rightarrow 0}{\underset{\approx}{\longrightarrow}} \varepsilon(\ln \varepsilon)^{z^{\prime}} \operatorname{const} \underset{\varepsilon \rightarrow 0}{\longrightarrow} 0 .
\end{gathered}
$$

Consider now the second term $\Pi^{(k)}\left(-m^{2} ; m, \mu\right)$ in (4.13a) and (4.13b). Again with the help of Weinberg's theorem we obtain $\left.l_{\sigma} \geqq 0, \sum_{j=1}^{l_{\sigma}} k_{j}<k-1\right)$ :

$$
\begin{aligned}
& (m \varepsilon)^{2 l_{\sigma}} \prod_{j=1}^{l_{\sigma}} \tilde{a}^{\left(k_{j}\right)}(\varepsilon m / \mu) \mathscr{R}_{\Gamma_{(2, l \sigma)}}\left(-\varepsilon^{2} m^{2} ; 0, \ldots, 0 ; \varepsilon m, \mu\right) \underset{\varepsilon \rightarrow 0}{\approx} \\
& \text { const } \varepsilon^{2}(\ln \varepsilon)^{z} \mathscr{R}_{\Gamma_{(2, l \sigma)}}\left(-m^{2} ; 0, \ldots, 0 ; m, \mu / \varepsilon\right) \underset{\varepsilon \rightarrow 0}{\approx} \varepsilon^{2}(\ln \varepsilon)^{z^{\prime \prime}} \text { const } \underset{\varepsilon \rightarrow 0}{\longrightarrow} 0
\end{aligned}
$$

and, therefore, $\Pi^{(k)}\left(-m^{2} ; m, \mu\right)=O\left(m^{2}(\ln m / \mu)^{z}\right)$ which completes the proof of the lemma.

It is now clear that each diagram with an arbitrary number of $b \Delta_{1}$ insertions has a limit in $m \rightarrow 0$ to the corresponding one of the critical theory and at the same time each diagram with at least one $a \Delta_{0} \equiv m \tilde{a} \Delta_{0}$-insertion vanishes at the critical point :

$$
\begin{aligned}
& (\varepsilon m)^{2 l_{\sigma}} \prod_{j=1}^{l_{\sigma}} \tilde{a}^{\left(k_{j}\right)}(\varepsilon m / \mu) \mathscr{R}_{\Gamma_{\left(L_{n}, L_{\sigma}+l_{\sigma}\right)}}(\{p\},\{q\},\{0\} ; \varepsilon m, \mu) \underset{\varepsilon \rightarrow 0}{\approx} \\
& \operatorname{const}(\ln \varepsilon)^{z^{\prime \prime \prime}} \varepsilon^{3-1 / 2 L_{n}-L_{\sigma}-\underline{\operatorname{deg}}_{\varepsilon} \mathscr{R}_{T}} \underset{\varepsilon \rightarrow 0}{\longrightarrow} 0 .
\end{aligned}
$$

In (5.2) the rules for determining the asymptotic power:

$$
\operatorname{deg}_{\varepsilon} \mathscr{R}_{\Gamma_{\left(L_{n}, L_{\sigma}+l_{\sigma}\right)}}(\{p / \varepsilon\},\{q / \varepsilon\},\{0\} ; m, \mu / \varepsilon) \leqq\left[3-1 / 2 L_{n}-L_{\sigma}\right]-1
$$

were used (cf. [47], Sect. V).

In the low-temperature phase, quite analogously, each renormalized diagram with $L_{F}=0$ ( $L_{F}$ is the number of external blind $F$-lines) in the critical limit $f \rightarrow 0$ goes over into the corresponding one of the critical theory. Moreover, we have:

8 We use the notations:

$$
\mathscr{R}_{\Gamma_{\left(L_{n}, L_{\sigma}\right)}} \equiv \mathscr{R}_{\Gamma_{\left(L_{n}, L_{\sigma}\right)}}(\{p\} ;\{q\} ; m, \mu),\{p\} \equiv\left\{p_{1}, \ldots, p_{L_{n}}\right\},\{q\} \equiv\left\{q_{1}, \ldots, q_{L_{\sigma}}\right\}, \mathscr{R}_{\Gamma_{\left(L_{n}, L_{\sigma}\right)}^{\{P\}}} \equiv \mathscr{R}_{I}^{\{P\}}
$$


Lemma 5.2. $\lim _{f \rightarrow 0} b^{(k)}\left(f^{2} / \mu\right)=b^{(k)}(0)$, the latter coinciding with (4.16).

The statement of this lemma is a particular case of the following:

Proposition 5.3. Each graph of the low temperature phase with $L_{F}>0$ vanishes in the critical limit $f \rightarrow 0$.

Proof. First we consider an arbitrary $1 P I$ graph $\Gamma_{\left(L_{n}, L_{\sigma}\right)}$ with $L_{F}(\Gamma)=1$. As it was pointed out after (2.21), the corresponding factor $s^{1 / 2}$ moves to the left in the sum over $\Gamma$-forests for $\mathscr{R}_{L}$ with $\delta, \varrho$ changing to (integers): $\delta^{\prime}=\delta-\frac{1}{2}, \varrho^{\prime}=\varrho-\frac{1}{2}$. Hence, an external $F$-line can be treated as an external $n_{F}$-line with zero momentum. Making use of the power counting rules [47] we obtain in the limit $f \rightarrow 0$ :

$$
\varepsilon^{1 / 2} f \mathscr{R}_{\Gamma_{\left(L_{n}+1, L_{\sigma}\right)}}\left(\{p\}, 0,\{q\} ; \varepsilon f^{2}, \mu\right) \underset{\varepsilon \rightarrow 0}{\approx} \operatorname{const} \varepsilon^{1 / 2}(\ln \varepsilon)^{z} \underset{\varepsilon \rightarrow 0}{\longrightarrow} 0 .
$$

Now consider $\Gamma_{\left(L_{n}, L_{\sigma}\right)}$ with $L_{F}=2$. For every $1 P I$ graph $\lambda \subset \Gamma_{\left(L_{n}, L_{\sigma}\right)}$ containing only one vertex $\mathbf{V}\left[\left(n, F s^{1 / 2}\right) \sigma\right]$ the corresponding factors $s^{1 / 2}$ "commute" with the subtraction operators $\tau_{\lambda}$ according to (2.21), and for all $\gamma \subseteq \Gamma_{\left(L_{n}, L_{\sigma}\right)}$ containing both vertices $\mathbf{V}\left[\left(n, F s^{1 / 2}\right) \sigma\right]$ we apply the $\mathrm{ZI}$ :

$$
\tau_{\gamma}^{\delta(\gamma), \varrho(\gamma)} S=S \tau_{\gamma}^{\delta(\gamma)-1, \varrho(\gamma)-1}+D_{p^{\gamma}, s^{\gamma}-1}^{\varrho(\gamma)-1}\left(1-\tau_{\gamma}^{\delta(\gamma)-1}, \varrho(\gamma)-1\right) .
$$

Thus we have $\left[\mathscr{Q}_{2}, \mathscr{Q}_{4}\right.$ - the same as in (4.5)]:

$$
\begin{aligned}
& \mathscr{R}_{\Gamma_{\left(L_{n}, L_{\sigma}\right)}}=f^{2}\left[\mathscr{R}_{\Gamma_{\left(L_{n}, 2, L_{\sigma}\right)}}+\left.\sum_{\gamma_{(4,0)} \subset \Gamma_{\left(L_{n}, L_{\sigma}\right)}}\left(\frac{\partial}{\partial S} \mathscr{R}_{\left.\gamma_{(4,0)}\right)}\right)\right|_{\substack{p^{\gamma} \gamma=0 \\
s^{\gamma}=1}} \mathscr{R}_{\Gamma_{\left(L_{n}, L_{\sigma}\right)} / \gamma_{(4,0)}}^{2_{4}}\right] \\
& +\sum_{\gamma_{(6,0)} C \Gamma_{\left(L_{n}, L_{\sigma}\right)}} \mathscr{R}_{\left.\gamma_{(6,0)}\right|_{s^{\gamma}=1} ^{p^{\gamma}=0}} \mathscr{R}_{\Gamma_{\left(L_{n}, L_{\sigma}\right) / \gamma_{(6,0)}}^{\mathscr{Q}_{2}}} .
\end{aligned}
$$

In (5.4) $\Gamma_{\left(L_{n}+2, L_{\sigma}\right)}^{0}$ is topologically equivalent to $\Gamma_{\left(L_{n}, L_{\sigma}\right)}$ whose external $F$-lines are considered as external $n_{F}$-lines at zero momenta, and all subtraction indices $\delta(\lambda)$, $\varrho(\lambda), \lambda \subseteq \Gamma_{\left(L_{n}+2, L_{\sigma}\right)}^{0}$ correspond to the canonical choice (2.20). The sums in the r.h.s. of (5.4) run over all subgraphs of $\Gamma_{\left(L_{n}, L_{\sigma}\right)}$ containing both external $F$-lines. Using the identity: $\partial / \partial s \tau^{\delta, \varrho}=\tau^{\delta-1, \varrho-1} \partial / \partial s$, the forest formula $(2.8)$ and the explicit expressions for the $n$ - and $\sigma$-propagators (A.4) and (A.5), we obtain:

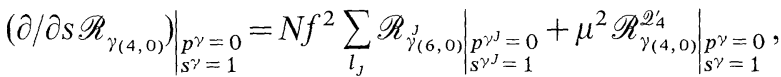

$$
\begin{aligned}
& \mathscr{Q}_{4}^{\prime}=\int d^{3} x \cdot \mathscr{N}_{2}^{2}\left[(1-s) n^{2}\right]_{\otimes}(x) \text {, }
\end{aligned}
$$

where the sum in the r.h.s. of (5.5) runs over all lines $l_{j} \in \mathscr{L}_{\sigma}\left(\gamma_{(4,0)}\right)$, in which the aggregate: $N f^{2} \mathscr{G}^{-1}\left(l_{j}^{2} ; m^{2}(s), s f^{2}\right) \times \mathscr{D}\left(l_{j}^{2} ; m^{2}(s)\right) \mathscr{G}^{-1}\left(l_{j}^{2} ; m^{2}(s), s f^{2}\right)$ is inserted (see Fig. 5). As a result of the ZI (4.7) the last term in (5.5) acquires the form:

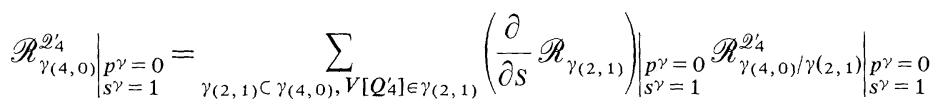

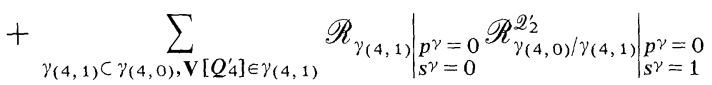




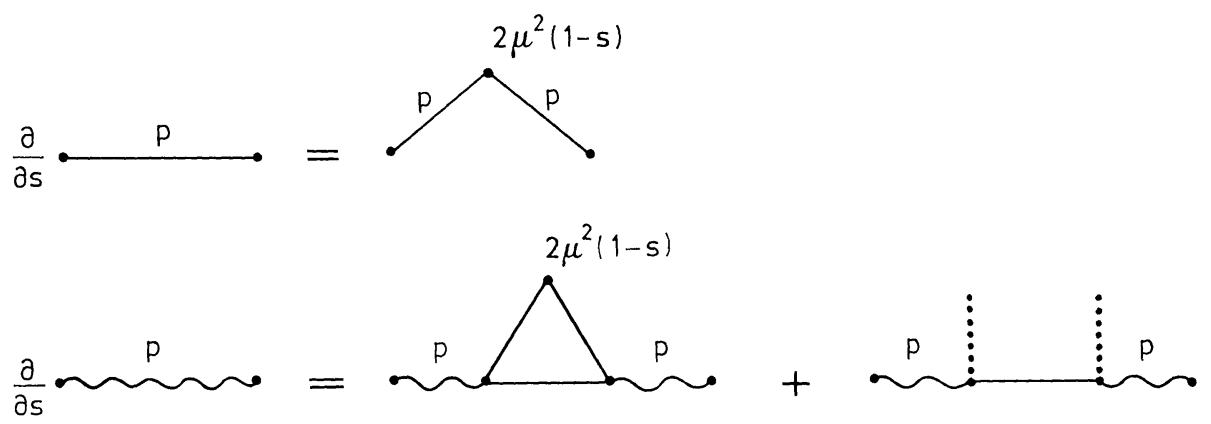

Fig. 5. Differentiation of $\mathscr{D}\left(p^{2} ; m^{2}(s)\right)$ and $\mathscr{G}\left(p^{2} ; m^{2}(s), s f^{2}\right)$ in $s$

and, finally, it reduces to graphs with $\mathscr{Q}_{2}^{\prime} \equiv \int d^{3} x \mathscr{N}_{2}^{2}\left[\left(n^{2}\right)^{2}\right]_{\otimes}(x)$-insertion. Note, that all coefficients in the square brackets in the r.h.s. of (5.6) remain finite when $f \rightarrow 0$ because of the substitution $s=0$.

Now applying Weinberg's theorem to all terms in the square brackets of the r.h.s. of (5.4) and taking into account (5.5) and (5.6) we find that they all have logarithmic asymptotae: $O\left(\left(\ln f^{2} / \mu^{z}\right)\right)$ and that is why the whole $\mathscr{R}_{\Gamma_{\left(L_{n}, L_{\sigma}\right)}}$ (with $L_{F}=2$ ) vanishes at the critical point.

The proof for $L_{F} \geqq 3$ proceedes exactly along the same lines by reducing $\Gamma_{\left(L_{n}, L_{\sigma}\right)}$ to a graph without external $F$-lines with the help of successive application of (5.3) and using Weinberg's theorem afterwards.

\section{RG Equations and WTIs for Broken Scale Invariance}

In the derivation of RG equations and WTIs for broken scale invariance in the high-temperature [Callan-Symanzik equations-(CS)] and in the low-temperature ("Goldstone-limit" equations (GL), cf. [45]) phases we shall follow the method of differential vertex operations [48]. The standard scheme is based on: (1) renormalized action principle, (2) integrated bilinear equations of motion, (3) Zimmermann identities. For the chiral field model one has to add: (4) integrated quantum chirality identities.

Proposition 6.1. The renormalized action principle in both phases as well as at the critical point holds in the following form:

$$
\begin{aligned}
& \left\{\mu \frac{\partial}{\partial \mu}-\mu \frac{\partial a}{\partial \mu} \Delta_{0}-\mu \frac{\partial b}{\partial \mu} \Delta_{1}-2 \mu m \mathscr{Q}_{4}-2 \mu(\mu-m) \mathscr{Q}_{5}\right\}\left\langle X_{n} X_{\sigma}\right\rangle=0, \\
& \left\{m \frac{\partial}{\partial m}-m \frac{\partial a}{\partial m} \Delta_{0}-m \frac{\partial b}{\partial m} \Delta_{1}-2 m^{2} \mathscr{Q}_{1}-2 m(\mu-m) \mathscr{Q}_{4}\right. \\
& \left.\quad+2 m(\mu-m) \mathscr{Q}_{5}\right\}\left\langle X_{n} X_{\sigma}\right\rangle=0, \\
& \left\{f^{2} \frac{\partial}{\partial f^{2}}-f^{2} \frac{\partial b}{\partial f^{2}} \Delta_{1}+\frac{1}{2} f \Delta_{3}\right\}\left\langle X_{n} X_{\sigma}\right\rangle=0,
\end{aligned}
$$

where $f \Delta_{3} \equiv \int d^{3} x \mathscr{N}_{3}^{3}\left[\sigma\left(n, F s^{1 / 2}\right)\right](x)$. 


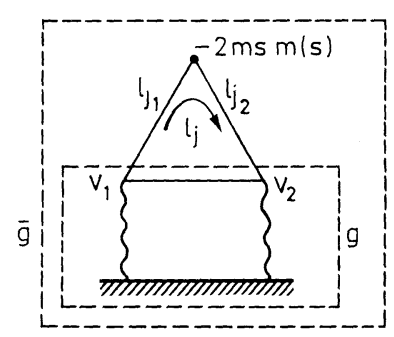

Fig. 6. Additional forests arising in the proof of the action principle

The proof is based on standard graphical arguments and the forest formula (2.8) [48]. We take, for instance, (6.1b) and consider an arbitrary connected graph $G$ of $\left\langle X_{n} X_{\sigma}\right\rangle$ with $k_{a}, k_{b}$ insertions of $a \Delta_{0}, b \Delta_{1}$, respectively. From (2.8) we have:

$$
\begin{aligned}
m \frac{\partial}{\partial m} R_{G}= & (-1)^{|\mathscr{V}(G)|+\left|\mathscr{L}^{\sigma}(G)\right|} N^{-\left|\mathscr{L}_{\sigma}(G)\right|}\left\{\left[S_{G} \sum_{U \in \mathscr{F}_{G}} \prod_{\lambda \in U}\left(-\tau_{\lambda} S_{\lambda}\right) \prod_{\mathscr{L}_{n}(G)} \mathscr{D}\left(l_{i}^{2} ; m^{2}(s)\right)\right.\right. \\
& \left.\cdot \prod_{\mathscr{L}_{\sigma}(G)} \mathscr{G}\left(l_{j}^{2} ; m^{2}(s)\right)\right]\left(k_{a} m \frac{\partial a}{\partial m} a^{k_{a}-1} b^{k_{\tilde{b}}}+k_{b} m \frac{\partial b}{\partial m} a^{k_{a}} b^{k_{b}-1}\right) \\
& +a^{k_{a}} b^{k_{b}}\left[\sum_{\mathscr{L}_{n}(G)}(-2 \sin m(s)) \mathscr{D}\left(l_{i}^{2} ; m^{2}(s)\right)\right. \\
& \left.\left.+\sum_{\mathscr{L}_{\sigma}(G)}\left(-N m \frac{\partial}{\partial m} \mathscr{S}\left(l_{j}^{2} ; m^{2}(s)\right)\right) \mathscr{G}\left(l_{j}^{2} ; m(s), 0\right)\right]\right\} .
\end{aligned}
$$

The first term in the parenthesis of (6.2) gives rise to $-m \frac{\partial a}{\partial m} \Delta_{0}-m \frac{\partial b}{\partial m} \Delta_{1}$ in (6.1b), and the terms $(-2 s m m(s))\left[\mathscr{D}\left(l_{i}^{2} ; m^{2}(s)\right)\right]^{2}$ represent a part of the corresponding graphs with $\mathscr{Q}_{1}, \mathscr{Q}_{4}, \mathscr{Q}_{5}$ insertions in $(6.1 \mathrm{~b})$. The remaining part of the needed graphs is obtained by analyzing the last sum in the r.h.s. of (6.2). Indeed, representing $m \partial / \partial m \mathscr{S}\left(l_{j}^{2} ; m^{2}(s)\right)$ as a triangle subdiagram we observe that in order to get the correct $\mathscr{2}_{1}, \mathscr{2}_{4}, \mathscr{2}_{5}$ insertions we have to add now forests, containing elements $g$ with the property: $(*) \mathbf{V}_{c} \in \mathscr{V}(g), l_{j, c} \notin \mathscr{L}(g), c=1,2$ (see Fig. 6). The set of all relevant forests reads: $\mathscr{F}_{G} \bigcup_{g}\left\{\mathscr{F}_{G}(g)\right\}$, where $\mathscr{F}_{G}(g)$ is the set of forests containing at least one element with property $(*)$ and $g$ is the maximal one among the latter (cf. the proof of Lemma 3.2). To each such $g$ we relate $\bar{g}=g \cup\left\{l_{j, c}\right\}_{c=1}^{2}$ (see Fig. 6) and denote the subset of $\mathscr{F}_{G}(g)$ in which all forests contain the corresponding $\bar{g}$ by $\mathscr{F}_{G}^{\prime}(g)$. Thus, the contribution of all new added forests $\bigcup_{g} \mathscr{F}_{G}(\mathrm{~g})$ to $R_{G}$ is :

$$
S_{G} \sum_{g} \sum_{U \in \mathscr{F}_{G}^{\prime}(g)} \prod_{\substack{\lambda(\bar{g}=\emptyset \\ \lambda \supset \bar{g}}}\left(-\tau_{\lambda} S_{\lambda}\right) I_{G / \bar{g}}\left\{\left(1-\tau_{\bar{g}}\right) S_{\bar{g}}\left(-2 m s m(s) \mathscr{D}_{1} \mathscr{D}_{2}\right)\left(-\tau_{g} \bar{R}_{g}\right)\right\},
$$

where $\mathscr{D}_{1,2}$ are the propagators associated with lines $l_{j, c}, c=1,2$ in Fig. 6 . According to $(2.20) \delta(\bar{g})=\delta(g)+1, \varrho(\bar{g})=\varrho(g)+1$ and that is why, in complete analogy with the case of Lemma 3.2 [cf. (3.4) and (3.5)], the expression in the parenthesis of (6.3) (after integration in the loop momentum $l_{j}$, Fig. 6) vanishes. 
The proof of (6.1a) is strictly analogous and that of (6.1c) is straightforward. The bilinear integrated quantum equations of motion [cf. (4.3)] look like:

$$
\begin{aligned}
& \left\{(1+b) \Delta_{1}+(\mu-m)^{2} \mathscr{Q}_{5}+2 m(\mu-m) \mathscr{Q}_{4}+m^{2} \mathscr{Q}_{1}+\Delta_{2}+\frac{1}{2} f \Delta_{3}\right. \\
& \left.\quad+\frac{1}{2} L_{n}\right\}\left\langle X_{n} X_{\sigma}\right\rangle=0 .
\end{aligned}
$$

Taking into account Theorem 4.1 and $\left(4.10 \mathrm{a}^{\prime}\right)$, in the high-temperature phase $(f \equiv 0)(6.1 \mathrm{a}),(6.1 \mathrm{~b})$, and (6.4) give three equations for two linearly independent vertex insertions $\Delta_{0}, \Delta_{1}$, and in the low-temperature phase $(m \equiv 0)(6.1 \mathrm{a}),(6.1 \mathrm{c})$, and (6.4) lead to three equations for $\Delta_{1}, \Delta_{3}$. As a result, in the first case the $R G$ and $C S$ equations arise $\left(L_{n} \neq 0\right)$ :

$$
\begin{aligned}
& \left\{\mu \frac{\partial}{\partial \mu}+L_{n} \zeta_{n}(m / \mu)+L_{\sigma} \zeta_{\sigma}(m / \mu)\right\}\left\langle X_{n} X_{\sigma}\right\rangle=0, \\
& \left\{\mu \frac{\partial}{\partial \mu}+m \frac{\partial}{\partial m}+L_{n} \gamma_{n}(m / \mu)+L_{\sigma} \gamma_{\sigma}(m / \mu)-m \alpha(m / \mu) \Delta_{0}\right\}\left\langle X_{n} X_{\sigma}\right\rangle=0,
\end{aligned}
$$

and in the second case one obtains the RG and GL equations:

$$
\begin{aligned}
& \left\{\mu \frac{\partial}{\partial \mu}-2 \zeta_{n}\left(f^{2} / \mu\right) f^{2} \frac{\partial}{\partial f^{2}}+L_{n} \zeta_{n}\left(f^{2} / \mu\right)+L_{\sigma} \zeta_{\sigma}\left(f^{2} / \mu\right)\right\}\left\langle X_{n} X_{\sigma}\right\rangle=0 \\
& \left\{\mu \frac{\partial}{\partial \mu}+f^{2} \frac{\partial}{\partial f^{2}}+L_{n} \gamma_{n}\left(f^{2} / \mu\right)+L_{\sigma} \gamma_{\sigma}\left(f^{2} / \mu\right)+\left(\frac{1}{2}+\gamma_{n}\left(f^{2} / \mu\right)\right) f \Delta_{3}\right\}\left\langle X_{n} X_{\sigma}\right\rangle=0 .
\end{aligned}
$$

For the vertex functions $\Gamma^{\left(L_{n}, L_{\sigma}\right)}, L_{n} \geqq 2$, all Eqs. (6.5)-(6.7) are the same with the only change: $L_{n} \rightarrow-L_{n}, L_{\sigma} \rightarrow-L_{\sigma}$. Substituting in (6.5)-(6.7) the normalizations conditions (4.12), $(4,14)$, and (4.17) we obtain simple expressions for all coefficient functions, e.g., for the anomalous dimensions $\zeta_{n}$ and $\zeta_{\sigma}$ of the $n$ - and $\sigma$-fields:

$$
\begin{aligned}
& \zeta_{n}(m / \mu)=\left.\mu^{2}\left(\mu^{2}+m^{2}\right)^{-1}\left(1+\partial / \partial p^{2} \Gamma^{(2,0)}\right)\right|_{p_{2}=\mu^{2}}, \\
& 2 \zeta_{n}(m / \mu)+\zeta_{\sigma}(m / \mu)=\left.2 \mu^{2}\left(\partial / \partial \mu^{2} \Gamma^{(2,1)}\right)\right|_{p_{1}^{2}=p_{2}^{2}=p_{1}, p_{2}=\mu^{2}} .
\end{aligned}
$$

In complete analogy with the case of noncanonically soft mass renormalized $O(N)$-invariant $\left(\varphi^{2}\right)_{3}^{2}$ model [40] one obtains in the limit $m \rightarrow 0$ or $f \rightarrow 0$ :

$$
\begin{aligned}
& \zeta_{n}(m / \mu)-\gamma_{n}(m / \mu)=O\left(m(\ln m / \mu)^{z}\right), \quad \zeta_{\sigma}(m / \mu)-\gamma_{\sigma}(m / \mu)=O\left(m(\ln m / \mu)^{z}\right), \\
& m \alpha(m / \mu)=O\left(m(\ln m / \mu)^{z}\right), \\
& \zeta_{n}\left(f^{2} / \mu\right)-\gamma_{n}\left(f^{2} / \mu\right)=O\left(f\left(\ln f^{2} / \mu\right)^{z}\right), \quad \zeta_{\sigma}\left(f^{2} / \mu\right)-\gamma_{\sigma}\left(f^{2} / \mu\right)=O\left(f\left(\ln f^{2} / \mu\right)^{z}\right) .
\end{aligned}
$$

Therefore, in the critical limit all differential equations (6.5)-(6.7) coincide:

$$
\left\{\mu \frac{\partial}{\partial \mu}+L_{n} \zeta_{n}(0)+L_{\sigma} \zeta_{\sigma}(0)\right\}\left\langle X_{n} X_{\sigma}\right\rangle=0 \text {. }
$$

\section{Critical Behaviour and Universality of the Chiral Field}

In this section we shall describe briefly the critical behaviour of the chiral field in the framework of the soft mass BPHZL renormalization approach. 


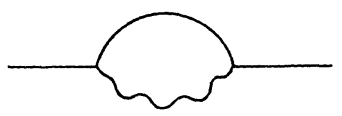

Fig. 7. Graphs contributing to $\eta^{(1)}$

The RG equations at the critical point $\left(6.5^{\prime \prime}\right)$ are just the dilatation WTIs $[46,40]$. In particular, the two point vertex function together with the normalization condition (4.16) acquires the well known scaling (conformally invariant) form:

$$
\Gamma^{(2,0)}\left(p^{2} ; \mu\right)=-\left(\mu^{2}\right)^{-\eta / 2}\left(p^{2}\right)^{1-\eta / 2}, \quad \eta \equiv 2 \zeta_{n}(0) .
$$

The evaluation of the critical exponent $\eta$ within the $1 / N$ expansion according to (6.8a) (for $m=0$ ) gives in the leading order (see Fig. 7) the answer: $\eta^{(1)}=8 / 3 \pi^{2} N$, which exactly coincides with the corresponding result for the $N$-component lattice Heisenberg model [36].

A characteristic feature of the BPHZL framework is that the physical normalization conditions (4.12) and (4.14) introduce as independent parameters the physical mass $m$ and the physical spontaneous magnetization $f$ in the high-and low-temperature phases, respectively, instead of the "temperature" $T$ itself. That is why, in order to establish all scaling laws and to prove the validity of the universality relations between critical exponents, one has to introduce "temperature" and "magnetic-field" perturbations on the effective Lagrangian of the critical theory $\left[\left(4.4^{\prime}\right)\right.$, with $\left.m=f=0\right]$ (cf. [34]):

$$
\mathscr{L}_{\text {eff }}(x ; \mu, t, H)=\mathscr{L}_{\text {eff }}(x ; 0,0, \mu)-N / 2 \mu t \sigma(x)+(H, n(x)) .
$$

Here $t\left(\sim\left(T-T_{c}\right) T_{c}^{-1}\right)$ describes small deviations from the critical temperature $T_{c}$. Of course, such supernormalizable perturbations do not exist as insertions in the conformally invariant chiral field Green's functions at the critical point, because of the IR divergencies, increasing with the number of insertions [cf. (2.2), (2.6), (2.12)]. We shall formulate a simple resummation procedure for (7.1), which does not change orders of $1 / N$, ending up with a well defined $1 / N$ expansion of the perturbed theory, free of IR divergencies in each separate diagram (cf. with the case of the soft mass noncanonically renormalized $O(N)$ invariant $\left(\varphi^{2}\right)_{4}^{2}$ model [40]).

For the sake of rigour, one first considers the "temperature" and "magnetic field" perturbation in the high-temperature phase, i.e.:

$$
\mathscr{L}_{\mathrm{eff}}(x ; m, \mu, t, H)=\mathscr{L}_{\mathrm{eff}}(x ; m, 0, \mu)-N / 2 \mu t \sigma(x)+(H, n(x))
$$

Following the standard procedure $[34,45]$ after the shifting: $n(x)=\bar{n}(x)+\tilde{F}$ $\left[\tilde{F}^{2} \equiv N \tilde{f}^{2}, \tilde{F}=\langle n(x)\rangle_{H, t}\right.$-true vacuum expectation value in the presence of the insertions $\left.\left.-N / 2 \mu t \int \sigma(x) d^{3} x, \int(H, n(x)) d^{3} x\right)\right]$, the new theory is reparametrized in terms of $\tilde{f}$ instead of $H$. The function $H=H(m, \mu, t, \tilde{f})$ is determined by the condition of absence of $\bar{n}$-tadpoles $\left(\langle\bar{n}(x)\rangle_{t, \tilde{f}}=0\right)$ recursively in orders of $1 / N$. The partial resummation of $\left(7.1^{\prime}\right)$, which does not change the orders of diagrams in 

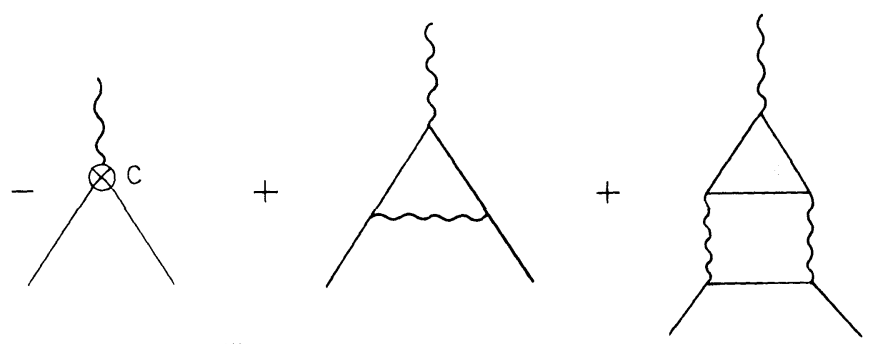

Fig. 8. Graphs contributing to $v^{(1)}$

$1 / N$, is described equivalently by the following effective Lagrangian $\left(m(t, f ; s) \equiv m(s)+4 \pi s\left(t \mu+f^{2}\right)\right)$ :

$$
\begin{aligned}
\hat{\mathscr{L}}_{\mathrm{eff}}(x ; m, \tilde{f}, \mu t)= & -\frac{1}{2}\left(1+\hat{b}\left(m / \mu, f^{2} / \mu, t\right)\right) \mathcal{N}_{3}^{3}\left[\left(\partial_{\mu} \bar{n}\right)^{2}\right]_{\otimes}(x)-\frac{1}{2} \mathcal{N}_{3}^{3}\left[m^{2}(t, \tilde{f} ; s) n^{2}\right]_{\otimes}^{*}(x) \\
& -\frac{1}{2} \cdot \mathcal{N}_{3}^{3}\left[\sigma\left(\bar{n}^{2}+2\left(n, F s^{1 / 2}\right)\right)\right]_{\otimes}^{*}(x)+N / 2 \hat{a}(m, \mu, t, \tilde{f}) \sigma(x) \\
& +\frac{1}{2} \cdot \mathcal{N}_{3}^{3}[\sigma(\tilde{\mathscr{G}}-1 \sigma)]_{\otimes}(x),
\end{aligned}
$$

where the new finite counterterms $\hat{a}, \hat{b}$ are explicitely determined as functions of the old ones $a, b$ (in the $1 / N$ expansion). Thus, the only problem which might arise in the limit $m \rightarrow 0$ is that of the IR finiteness of $\hat{a}(0, \mu, t, \tilde{f})$ and $\hat{b}\left(0, t, f^{2} / \mu\right)$.

The proof of the existence of $\lim _{m \rightarrow 0} \hat{b}\left(m / \mu, t, \tilde{f}^{2} / \mu\right)$ and $\lim _{m \rightarrow 0} \hat{a}(m, \mu, t, \tilde{f})$ is not difficult and we omit it here (for the soft mass renormalized $O(N)\left(\varphi^{2}\right)_{4}^{2}$ model see [40]).

Thus the physical mass $m_{\text {phys }}(\mu, t)$ corresponding to the "temperature"perturbed critical theory $(7.4)(m=\tilde{f}=0)$ is well defined and obeys the RG equation:

$$
\begin{aligned}
& \left\{\mu \frac{\partial}{\partial \mu}-\left(1-\zeta_{\sigma}(0)\right) t \frac{\partial}{\partial t}\right\} m_{\text {phys }}(\mu, t)=0, \\
& m_{\text {phys }}(\mu, t)=\text { const } \mu t^{\left(1-\zeta_{\sigma}(0)\right)^{-1}}
\end{aligned}
$$

wherefrom one obtains the second independent critical exponent $v=\left(1-\zeta_{\sigma}(0)\right)^{-1}$. Taking into account (6.8a) and (6.8b) (for $m=0)$ straightforward computation (see Fig. 8) (making use of, e.g., the methods for conformally invariant integration [50]) gives in the leading order of $1 / N: \zeta_{\sigma}^{(1)}(0)=v^{(1)}=-32 / 3 \pi^{2} N$, which exactly agrees with the corresponding calculations for the lattice classical Heisenberg model [36].

Exactly along the same lines one can consider "temperature"-perturbation of the Goldstone phase, i.e. deviations from the critical theory from below (when $f \rightarrow 0$ ). The corresponding effective Lagrangian, describing the partial resummation of the "temperature" insertion $\mu t \Delta_{0}$ looks like (7.2), but with other counterterms $\hat{a}^{\prime}(\mu, t, f), \hat{b}^{\prime}\left(f^{2} / \mu, t\right)$, which are IR finite. Here $f$ ceases to be the physical magnetization (for $t \neq 0$ ), unlike the case for $\tilde{f}$ in (7.2).

The simple proof of the existence of $\lim _{f \rightarrow 0} \hat{b}^{\prime}\left(f^{2} / \mu, t\right), \lim _{f \rightarrow 0} \hat{a}^{\prime}(\mu, t, f)$ proceeds in complete analogy with the case of $O(N)\left(\varphi^{2}\right)_{4}^{2}$ model [40] and is omitted. 


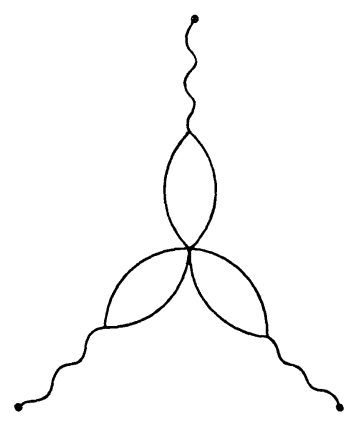

Fig. 9. Graph giving rise to contact terms in the chirality identity for $\int d^{3} x\left\langle\mathscr{N}_{3}^{3}\left[\left(n^{2}\right)^{3}\right](x) \sigma\left(x_{1}^{\prime \prime}\right) \sigma\left(x_{2}^{\prime \prime}\right) \sigma\left(x_{3}^{\prime \prime}\right)\right\rangle$

In particular, the physical magnetization $f_{\text {phys }}(\mu, t)$ for $f=0\left(F_{\text {phys }}=\langle n\rangle_{t}, F_{\text {phys }}^{2}\right.$ $=N f_{\text {phys }}^{2}$ ) is well defined and obeys the $\mathrm{RG}$ equation from which the critical exponent $\beta$ emerges :

$$
\begin{aligned}
& \left\{\mu \frac{\partial}{\partial \mu}-\left(1-\zeta_{\sigma}(0)\right) t \frac{\partial}{\partial t}+\zeta_{n}(0)\right\} f_{\text {phys }}(\mu, t)=0, \\
& f_{\text {phys }}(\mu, t)=\mathrm{const} \mu^{1 / 2} t^{\beta}, \quad \beta=\left(\frac{1}{2}+\zeta_{n}(0)\right)\left(1-\zeta_{\sigma}(0)\right)^{-1} \equiv \frac{1}{2} v(1+\eta) .
\end{aligned}
$$

From here on by means of standard technique $[34,50,46,38,51]$ based on the RG equations $\left(L_{n} \neq 0\right)$ :

$$
\begin{gathered}
\left\{\mu \frac{\partial}{\partial \mu}-\left(1-\zeta_{\sigma}(0)\right) t \frac{\partial}{\partial t}-\zeta_{n}(0)\left(L_{n}+2 \tilde{f}^{2} \frac{\partial}{\partial \tilde{f}^{2}}\right)-\zeta_{\sigma}(0) L_{\sigma}\right\} \\
\cdot \Gamma^{\left(L_{n}, L_{\sigma}\right)}(\{p\},\{q\} ; 0, \mu, t, \tilde{f})=0
\end{gathered}
$$

whose validity is proved rigorously in the present approach all scaling laws, e.g., the global Kadanoff's scaling [35]:

$$
\begin{aligned}
\Gamma^{\left(L_{n}, L_{\sigma}\right)}(\{\kappa p\},\{\kappa q\} ; 0, \mu, t, \tilde{f})= & \kappa^{3-L_{n}\left(1 / 2+\zeta_{n}(0)\right)-L_{\sigma}\left(2+\zeta_{\sigma}(0)\right)} \\
& \cdot \Gamma^{\left(L_{n}, L_{\sigma}\right)}\left(\{p\},\{q\} ; 0, \mu ; t \kappa^{-\left(1-\zeta_{\sigma}(0)\right)}, \tilde{f} \kappa^{-\left(1+\zeta_{n}(0)\right)}\right),
\end{aligned}
$$

and universality relations (for $D=3$ and $N$ components) among critical exponents are established to hold.

For instance, for the specific heat $C_{H=0}=\left.\operatorname{const}\left(\partial^{2} \partial t^{2} \mathscr{F}\right)\right|_{H=0}(\mathscr{F}$ : free energy), due to the arising of additive contact terms in the chirality identity (4.10b) (see Fig. 9) for $L_{n}=0, L_{\sigma}=3$ :

$$
\begin{aligned}
\int d^{3} x\left\langle\mathcal{N}_{3}^{3}\left[\left(n^{2}\right)^{3}\right]_{\otimes}(x) \sigma\left(x_{1}^{\prime \prime}\right) \sigma\left(x_{2}^{\prime \prime}\right) \sigma\left(x_{3}^{\prime \prime}\right)\right\rangle \\
=\sum_{i=1}^{3} d_{3, i}(m / \mu)\left\langle\Delta_{i} \sigma\left(x_{1}^{\prime \prime}\right) \sigma\left(x_{2}^{\prime \prime}\right) \sigma\left(x_{3}^{\prime \prime}\right)\right\rangle-\tilde{C}_{3}(m / \mu) \delta\left(x_{1}^{\prime \prime}-x_{2}^{\prime \prime}\right) \delta\left(x_{2}^{\prime \prime}-x_{3}^{\prime \prime}\right)
\end{aligned}
$$


one encounters an additional term linear in $t$ in the scaling law:

$$
C_{H=0}=\text { const } t^{-\alpha}-\frac{3 v C_{3}}{1+\alpha} t, \quad \alpha=2-3 v,
$$

where the constant $C_{3}$ is expressed by $\tilde{C}_{3}(0)$.

Acknowledgements. The authors are deeply indebted to L. D. Faddeev, I. T. Todorov, V. N. Popov, and V. E. Korepin for stimulating interest in this work. We also thank K. Symanzik and B. Schröer for kind correspondence.

Note Added. Prof. K. Symanzik has recently kindly informed us that he has a considerable amount of unpublished results of $1 / N$ expansion concerning massive $P\left(\varphi^{2}\right)_{D}(2 \leqq D \leqq 4)$ and chiral field $(2 \leqq D \leqq 4)$ models as well as computations of critical exponents $\eta$ (all $D$ ) and $v(D=3)$ to order $1 / N^{2}$.

\section{Appendix}

\section{Diagram Technique of $1 / N$ Expansion}

Here we briefly remind the diagram technique of $1 / N$ expansion $[21,24]$. The (unrenormalized) generating functional for the Euclidean Green's functions of the chiral field is:

$$
\begin{aligned}
Z(J) & =\mathrm{const} \int \prod_{x} d n(x) \delta\left(n^{2}(x)-\mu^{N} / T\right) \exp \int d^{3} x\left[-1 / 2\left(\partial_{\mu} n\right)^{2}+(J, n)\right] \\
& =\mathrm{const} \int \prod_{x} d n(x) d \sigma(x) \exp \int d^{3} x\left[-1 / 2\left(\partial_{\mu} n\right)^{2}-1 / 2 \sigma\left(n^{2}-\mu^{N} / T\right)+(J, n)\right] \\
& =\mathrm{const} \int \prod_{x} d n_{F}(x) d \sigma(x) \exp \left\{N A_{1}\left[\sigma, n_{F}\right]+A_{2}\left[\sigma, n_{F} ; J\right]\right\}
\end{aligned}
$$

where in the last line the following notations are used $\left(\hat{F}\right.$-fixed vector on $\left.\mathbf{S}^{N-1}\right)$ :

$$
\begin{aligned}
& n=n_{\perp}+\sqrt{N} n_{F} \hat{F}, \quad J=J_{\perp}+J_{F} \hat{F}, \quad\left(n_{\perp}, \hat{F}\right)=\left(J_{\perp}, \hat{F}\right)=0, \quad \sqrt{N} n_{F}=(n, \hat{F}), \\
& J_{F}=(J, \hat{F}), \quad \hat{F}^{2}=1, \\
& \quad A_{1}\left[\sigma, n_{F}\right]=-1 / 2 \operatorname{Tr} \ln (-\square+\sigma)-1 / 2 \int d^{3} x\left[\left(\partial_{\mu} n_{F}\right)^{2}+\sigma\left(n_{F}^{2}-\mu / T\right)\right], \\
& A_{2}\left[\sigma, n_{F} ; J\right]=1 / 2 \operatorname{Tr} \ln (-\square+\sigma)+1 / 2 \int d^{3} x d^{3} y J_{\perp}(x)(-\square+\sigma)^{-1}(x, y) J_{\perp}(y) \\
& +\sqrt{N} \int d^{3} x J_{F}(x) n_{F}(x),
\end{aligned}
$$

and the integration over $n_{\perp}(x)$ has been taken.

$1 / N$ expansion of (A.1) is equivalent to the expansion around constant saddle points of $A_{1}\left[\sigma, n_{F}\right]: \sigma^{(c)} \equiv m^{2}$ and $n_{F}^{(c)} \equiv f$. The equations for these are:

$$
\begin{aligned}
& \mu / T-f^{2}-\mathscr{D}\left(0 ; m^{2}\right)=0, \quad m^{2} f=0, \\
& \mathscr{D}\left(x ; m^{2}\right)=\int \frac{d^{3} p}{(2 \pi)^{3}} \frac{e^{i p x}}{m^{2}+p^{2}}=\int \frac{d^{3} p}{(2 \pi)^{3}} e^{i p x} \mathscr{D}\left(p^{2} ; m^{2}\right) .
\end{aligned}
$$

The first Eq. (A.2) is not well-defined. To give sense to it we replace $\mathscr{D}\left(0 ; m^{2}\right)$ by the renormalized with "soft" mass and minimal subtractions $\mathscr{D}^{\text {ren }}\left(0 ; m^{2}(s)\right) 0<s<1$ 
$m(s) \equiv s m+(1-s) \mu:$

$\mathscr{D}^{\mathrm{ren}}\left(0 ; m^{2}(s)\right)=\int \frac{d^{3} k}{(2 \pi)^{3}}\left(1-t_{s}^{0}\right)\left(m^{2}(s)+k^{2}\right)^{-1}=s(\mu-m) / 4 \pi+a_{0} \mu / 4 \pi$,

where $a_{0}$ is an arbitrary constant accounting for the ambiguity of the subtraction. The renormalized thus Eq. (A.2) have three types of solutions:

1. $O(N)$-symmetric (high-temperature) phase solution: $T>T_{c}, f \equiv 0$, $m=4 \pi \mu\left(T_{c}^{-1}-T^{-1}\right), T_{c} \equiv 4 \pi\left(a_{0}+1\right)^{-1}$.

2. Spontaneously broken symmetry (Goldstone, low-temperature) phase solution: $T<T_{c}, m \equiv 0, f^{2}=\mu\left(T^{-1}-T_{c}^{-1}\right)$.

3. Critical theory: $T=T_{c}, m \equiv f \equiv 0$.

We note that $a_{0}$ corresponds to a finite renormalization of the critical temperature $T_{c}$. The phase transition occurs in each order of $1 / N$. In 1) and 2) the "temperature" $T$ is transmutated to the parameters $m$ and $f$, respectively.

The graph elements are depicted in Fig. 1. The expressions for the $n$ - and $\sigma$-field propagators (with "soft" mass) $\mathscr{D}\left(p^{2} ; m^{2}(s)\right)$ and $\mathscr{G}\left(p^{2} ; m(s), s f^{2}\right)$ are:

$$
\begin{aligned}
\mathscr{D}\left(p^{2} ; m^{2}(s)\right) & =\left(m^{2}(s)+p^{2}\right)^{-1}, \\
\mathscr{S}\left(p^{2} ; m^{2}(s), s f^{2}\right) & =\left[\mathscr{S}\left(p^{2} ; m^{2}(s)\right)+s f^{2} \mathscr{D}\left(p^{2} ; m^{2}(s)\right)\right]^{-1}, \\
\mathscr{S}\left(p^{2} ; m^{2}(s)\right) & =1 / 2 \int \frac{d^{3} k}{(2 \pi)^{3}}\left\{\left(m^{2}(s)+k^{2}\right) /\left(m^{2}(s)+(p-k)^{2}\right)\right. \\
& =(8 \pi|p|)^{-1} \operatorname{arctg} \frac{|p|}{2 m(s)} .
\end{aligned}
$$

The asymptotic behaviour of the propagators (A.4) and (A.5) is characterized by the upper $\left(\overline{\mathrm{deg}}_{p, s}\right)$ and lower $\underline{\operatorname{deg}}_{p, s-1}$ degrees [43]:

$$
\overline{\operatorname{deg}}_{p . s} \mathscr{D}=\underline{\operatorname{deg}}_{p, s-1} \mathscr{D}=-2, \quad \overline{\operatorname{deg}}_{p, s} \mathscr{G}=1, \quad \underline{\operatorname{deg}}_{p, s-1} \mathscr{G}=\left\{\begin{array}{l}
2 \\
1
\end{array},\right.
$$

where $\operatorname{deg}_{p, s-1}$ refers to the Goldstone phase and the critical theory, respectively.

Figure 1b lists the (sub)graphs "forbidden" in the diagrams for the Green's functions without composite operator insertions $Q(n, \sigma)$. However, they are allowed when $Q(n, \sigma)$ containing either of $n^{2}, \sigma n^{2}$ or $\sigma\left(n, \hat{F} S^{-1 / 2}\right)$ are present.

\section{References}

1. Stanley, H.E.: Phase transitions and critical phenomena. Oxford: Cambridge University Press 1971

2. Weinberg, S.: Phys. Rev. Lett. 18, 188 (1967)

3. Faddeev, L.D.: Dokl. Acad. Nauk SSSR 210, 807 (1973) (in Russian)

4. Slavnov, A.A.: Theor. Math. Phys. 10, 305 (1972) (in Russian)

5. Skyrme, T.R.H.: Proc. Roy, Sec. A247, 260 (1958); J. Math. Phys. 12, 1735 (1971)

6. Faddeev, L.D. : In : Nonlinear, nonlocal, and nonrenormalizable field theories. Dubna, JINR 1976 (in Russian) 
7. Belavin, A.A., Polyakov, A.M.: Nucl. Phys. B123, 429 (1977)

Förster, D.: Nucl. Phys. B 130, 38 (1977)

8. Polyakov, A.M.: Phys. Lett. B59, 79 (1975);

Migdal, A.A.: JETP Lett. 69, 1457 (1975) (in Russian)

9. Bardeen, W.A., Lee, B.W., Shrock, R.E.: Phys. Rev. D14, 985 (1976)

10. Brezin, E., Zinn-Justin, J.: Phys. Rev. D 14, 2615 (1976)

11. Aref'eva, I.Ya.: Theor. Math. Phys. 29, 147 (1976) (in Russian)

12. Aref'eva, I.Ya.: Theor. Math. Phys. 36, 24 (1978) (in Russian)

13. Polyakov, A.M.: Phys. Lett. B72, 224 (1977)

14. Aref'eva, I.Ya., Kulish, P.P., Nissimov, E.R., Pacheva, S.J.: LOMI preprint, E-1-1978

15. Lüscher, M.: Nucl. Phys. B135, 1 (1978)

16. Zamolodchikov, A.B., Zamolodchikov, Al.B. : Nucl. Phys. B132, 48 (1977)

17. Witten, E.: Phys. Rev. D16, 2991 (1977)

Di Vecchia, P., Ferrara, S. : Nucl. Phys. B130, 93 (1977)

Alvarez, O.: Phys. Rev. D 17, 1123 (1978)

Aref'eva, I.Ya., Krivoschekov, V.K., Medvedev, P.B. : ITEP preprint, 173 (1978)

18. Bogoliubov, N.N., Shirkov, D.V.: Introduction to the theory of quantized fields. New York: Interscience 1959

Hepp, K.: Theorie de la renormalization. Berlin, Heidelberg, New York: Springer 1969

19. Brezin, E., Zinn-Justin, J.: Phys. Rev. Lett, 36, 691 (1976); Phys. Rev. B14, 3110 (1976)

20. Hikami, S., Brezin, E.: J. Phys. A11, 1141 (1978)

21. Aref'eva, I.Ya.: Theor. Math. Phys. 31, 3 (1977) (in Russian)

22. Aref'eva, I.Ya.: In: Proc. Leningrad Dept. Steclov Math. Inst. (eds. P.P. Kulish, V. N.Popov) (in Russian) 77, 3 (1978)

23. Aref'eva, I.Ya.: Ann. Phys. (N.Y.) 117, 393 (1979)

24. Aref'eva, I.Ya.: Theor. Math. Phys. 36, 159 (1978) (in Russian)

25. Lowenstein, J., Zimmenmann, W.: Nucl. Phys. B86, 77 (1975); Commun. Math. Phys. 46, 105 (1976)

26. Lowenstein, J.: Commun. Math. Phys. 47, 53 (1976)

27. Lowenstein, J.: Nucl. Phys. B96, 189 (1975)

28. Amati, D., Rouet, A.: CERN preprint TH.2468 (1978)

29. Clark, T.: Max Planck Inst. preprint MPI-PAE/Pht 43/75

Clark, T., Rouet, A. : MPI-PAE/Pth 17/75

30. Zimmermann, W.: In: Lectures on elementary particles and quantum field theory, Vol. 1, p. 397 (eds. S. Deser, M. Grisaru, H.Pendleton). Cambridge: M.I. Press 1970

Zimmermann, W.: Ann. Phys. (N.Y.) 77, 536, 569 (1973)

31. Anikin, S.A., Polivanov, M.C., Zavialov, O.I.: Fortschr. Phys. 27, 459 (1977)

32. Lowenstein, J.: Phys. Rev. D4, 2281 (1971)

33. Lam, Y.M.P.: Phys. Rev. D6, 2145 (1972)

Gomes, M., Lowenstein, J.: Phys. Rev. D7, 550 (1973)

34. Jegerlehner, F., Schröer, B.: Acta Phys. Austriaca, Suppl. XI, 389 (1973)

35. Kadanoff, L.: Physics 2, 263 (1966)

Kadanoff, L., Götze, W., Hamblen, D., Hecht, R., Lewis, E., Palciauskas, V., Raul, B., Swift, J.: Rev. Mod. Phys. 39, 395 (1967)

36. Abe, R.: Prog. Theor. Phys. 48, 1414 (1972) Abe, R., Hikami, S.: Phys. Lett. A42, 419 (1973)

Prog. Theor. Phys. 49, 442 (1973)

37. Ma, S.: Rev. Mod. Phys. 45, 589 (1973); Wilson, K., Kogut, J.: Phys. Rep. C12, 75 (1974)

38. Brezin, E., Le Guillou, J.G., Zinn-Justin, J.: In: Phase transitions and critical phenomena (eds. C. Comb, M. Green). New York: Academic Press 1976

39. Symanzik, K.: DESY preprint $77 / 05$

40. Nissimov, E.R., Pacheva, S.J.: Theor. Math. Phys. 41, 55, 220 (1979) (in Russian)

41. Zimmermann, W.: Commun. Math. Phys. 15, 208 (1969)

42. Clark, T., Lowenstein, J.: Nucl. Phys. B113, 109 (1976)

43. Lowenstein, J., Zimmermann, W.: Commun. Math. Phys. 44, 73 (1975)

44. Gomes, M., Lowenstein, J., Zimmermann, W.: Commun. Math. Phys. 39, 81 (1974) 
45. Jegerlehner, F., Schröer, B. : Nucl. Phys. B68, 461 (1974)

46. Jegerlehner, F.: Fortschr. Phys. 23, 71 (1975)

47. Weinberg, S. : Phys. Rev. 118, 838 (1960)

48. Lowenstein, J.: Commun. Math. Phys. 24, 1 (1971)

49. Symanzik, K.: Lett. Nuovo Cimento 3, 734 (1972)

50. Di Castro, C., Jona-Lasinio, G., Peliti, L.: Ann. Phys. N.Y. 87, 327 (1974)

51. Brezin, E.: In : Methods in field theory (eds. R. Balian, J. Zinn-Justin). Amsterdam: North-Holland 1976

Communicated by E. Brezin

Received June 13, 1979 